\title{
Plants in alcoholic beverages on the Croatian islands, with special reference to rakija travarica
}

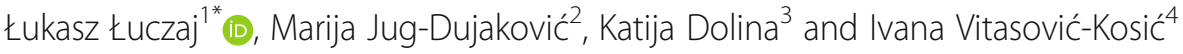

\begin{abstract}
Background: This paper aims to record the species used for flavouring and making alcoholic drinks, mainly rakija, on the islands of the Adriatic (Croatia).

Methods: Our data comes from 295 interviews performed on 36 islands, in both the Dalmatian and Kvarner areas of the Adriatic.

Results: Altogether, 114 species are used-46\% from wild locations only, 15\% both wild and cultivated, 38\% only cultivated and two species are imported.

The most common local alcohol is wine, made without spices, but grape pomace distillate is often flavoured with single or mixed species. The mix is called travarica. The most commonly used species are Foeniculum vulgare Mill., Myrtus communis L., Salvia officinalis L., Ruta graveolens L., Juniperus oxycedrus L., Ceratonia siliqua L., Juglans regia L., Citrus spp., Ficus carica L., Laurus nobilis L., Rosmarinus officinalis L., Artemisia absinthium L., Rosa centifolia L., Mentha $\times$ piperita L. and M. spicata L. Unfortunately, the widespread phenomenon of distilling Arbutus unedo L. fruits and fermenting Juniperus 'wine' is now extinct. Apart from grapes, the only commonly distilled fruit now is Ficus carica.

Conclusions: It is striking that nearly all the plants are either wild or cultivated locally, which, in addition to the fact that the alcohol is made locally, shows the incredible local culinary self-sufficiency of the area. The number of species used is also very impressive.
\end{abstract}

Keywords: Travarica, Ethnobotany, Culinary independence, Alcohol, Grappa, Mediterranean cuisine

\section{Background}

Regional alcoholic drinks are an important part of local culinary identity, not only in Europe [1-15]. In some parts of Europe, this identity is associated with local wines; in others, it is beer or high-percentage alcohol. Plants used for flavouring alcoholic drinks in some parts of Europe have been hardly documented, a rare example being Tuscany, Italy $[1,2]$.

It is worth mentioning that alcoholic drinks should not only be seen as intoxicating beverages or gourmet products, but also as components of local food security strategies, as pointed out by Madej et al. [9]. Excess fruit, when turned into an alcoholic drink, can be stored for a

\footnotetext{
* Correspondence: lukasz.luczaj@interia.pl

${ }^{1}$ Institute of Biology and Biotechnology, University of Rzeszów, ul. Pigonia 1, 35-310 Rzeszów, Poland

Full list of author information is available at the end of the article
}

long period of time in its new form. Additionally, the drink preserves some of the caloric value or other nutritional qualities of the original product from which it derives. Moreover, flavoured alcoholic drinks are strongly embedded in the tradition of herbalism. Alcohol is a very good material for the chemical extraction of medicinally active chemicals; hence, its use for making tinctures has been widespread since antiquity [14-17].

The coast of Croatia has recently been the subject of intense ethnobotanical investigation [18-27], mainly by the authors of this paper [18-25].

Most of the studies were focused on wild foods, though medicinal plant use in Istria and inland Dalmatia was also recorded. Wine is the dominant locally produced alcoholic drink in Croatia, hence a large variety of grape cultivars and types of wine are known. After winemaking, the remaining pressed grape stalks and pulp (i.e. 
pomace) are distilled into a grappa-type spirit called rakija.

Drinking rakija is also a social phenomenon. It is nearly always offered to guests and is drunk on many important occasions such as weddings and funerals. It is also consumed as a simple digestive. Rakija is made in nearly every rural household in coastal Croatia. However, since joining the European Union in 2013, there have been constraints on the selling of home-made spirits, and a special licence is now required.

Rakija is actually a term meaning all spirits which originate from the fermentation of fruits. In this paper, we will use the term rakija and grappa interchangeably. The word rakija comes from the Turkish raki (originally a word of Middle Eastern origin) and is commonly used not only in Croatia, but in other Balkan countries, which used to be a part of the Ottoman Empire [28-31]. On the other hand, it is argued that the making of rakija in the neighbouring Bosnia may have been brought from Dalmatia where spirits were made earlier than in the western part of the Ottoman Empire [31], the Venetians being pioneers of the production of aromatic spirits in Europe [15]. In Croatia, strong spirits are often flavoured with herbs and fruits. In spite of the fact that this tradition is widespread, as yet no monograph documents this phenomenon in its entirety. The only sources on flavoured rakija are a few popular handbooks documenting how to make it $[32,33]$. These however tend to contain species promoted by the author rather than documenting local practices or traditions. In order to fill this gap, we aimed to record the traditions of flavouring alcohol on the islands of the Croatian coast. Croatian islands are a good model for studying island biogeography principles [34] in ethnobotany. The theory of island biogeography [35] has hardly been applied at all in ethnobotany, our previous study on the use of wild vegetables on the Croatian islands constituting the first systematic study of this kind [34]. We found hardly any correlations between island geographical characteristics (such as size, population, flora and isolation from the mainland) and the number of wild vegetables used, apart from a generally increasing richness from the northwest to the southeast. However, in the previous paper, we only looked at the 15 largest islands, whereas in this study, we have data from nearly all the inhabited islands, including the most sparsely inhabited, presenting a greater variety of island sizes.

The main aim of our study was to compile a list of species used to flavour alcohol and determine whether there are any major differences within the study area. The islands of Croatia form one of the largest island archipelagos in Europe. We expected that the high diversity of dialects, relative isolation of local populations and abundance of aromatic Mediterranean plants would result in a high number of taxa used.

\section{Methods}

In the Croatian part of the Adriatic there are 1151 islands, islets and reefs, and 80 additional reefs which periodically appear above sea level, depending on the tides. Only 47 islands are inhabited, in the sense that at least one person resides there. Sixty Croatian islands have coastlines longer than $10 \mathrm{~km}$, while 653 islets have coastlines shorter than $10 \mathrm{~km}$, but with developed soil and vegetation. The highest elevation occurs on Brač (778 $\mathrm{m}$ a.s.l.). The climate of the area is mainly Mediterranean. According to the Köppen classification system, the coast and islands of the eastern Adriatic side belong to three Mediterranean climate types: (1) Cfsax, (2) Csax and (3) Csa. According to Mazzoleni et al., the entire area belongs to the IV3 climate type [36]. The potential vegetation over the largest areas of the studied islands is made up of forests dominated by Quercus ilex (belonging to the phytosociological class of Quercetea ilicis). On the Kvarner islands and the inland hills of larger islands, the potential natural vegetation is made up of downy oak forests (Quercetalia pubescentis) [37, 38].

The traditional food systems of the islands were based on utilising marine resources, mainly fish, and the cultivation of olives and grapes supplemented by grains, legumes and brassicas, along with animal husbandry, particularly in off-coast locations. Nowadays, the tourist industry is the dominant source of income.

This study forms part of a larger study on the ethnobotany of the Adriatic islands, from which only data about wild vegetable use has so far been published [34]. The research was performed between 2013 and 2018, with most interviews carried out in 2016 and 2017. One of the study questions enquired as to which plants were used to make flavoured rakija, liqueurs and other alcoholic drinks. The data in the spreadsheet come from 295 interviews (279 unpublished and 16 interviews from which data had already been published in an earlier paper about the island of Krk [20]). The mean age of respondents was 69 (median 70, minimum 30, maximum 96; $62 \%$ female, $38 \%$ male). We managed to interview people from 36 out of the 47 officially inhabited islands, including the 15 largest islands, with surface areas of over $40 \mathrm{~km}^{2}$ (Fig. 1, Table 1).

We applied the standard methods of ethnobotany: indepth semi-structured interviews starting from freelisting and supplemented if possible by walks around the places where the respondents gathered plants and could identify the supplied names. Two of the authors of this paper were brought up on the coast of Dalmatia (M.J.D. - Split; K.D. - Dubrovnik) and had frequently visited many of the studied islands since childhood. Some key informants were also selected by walks through the fields with people who claimed that they still collected wild food plants or made well-flavoured rakija. The interviews 


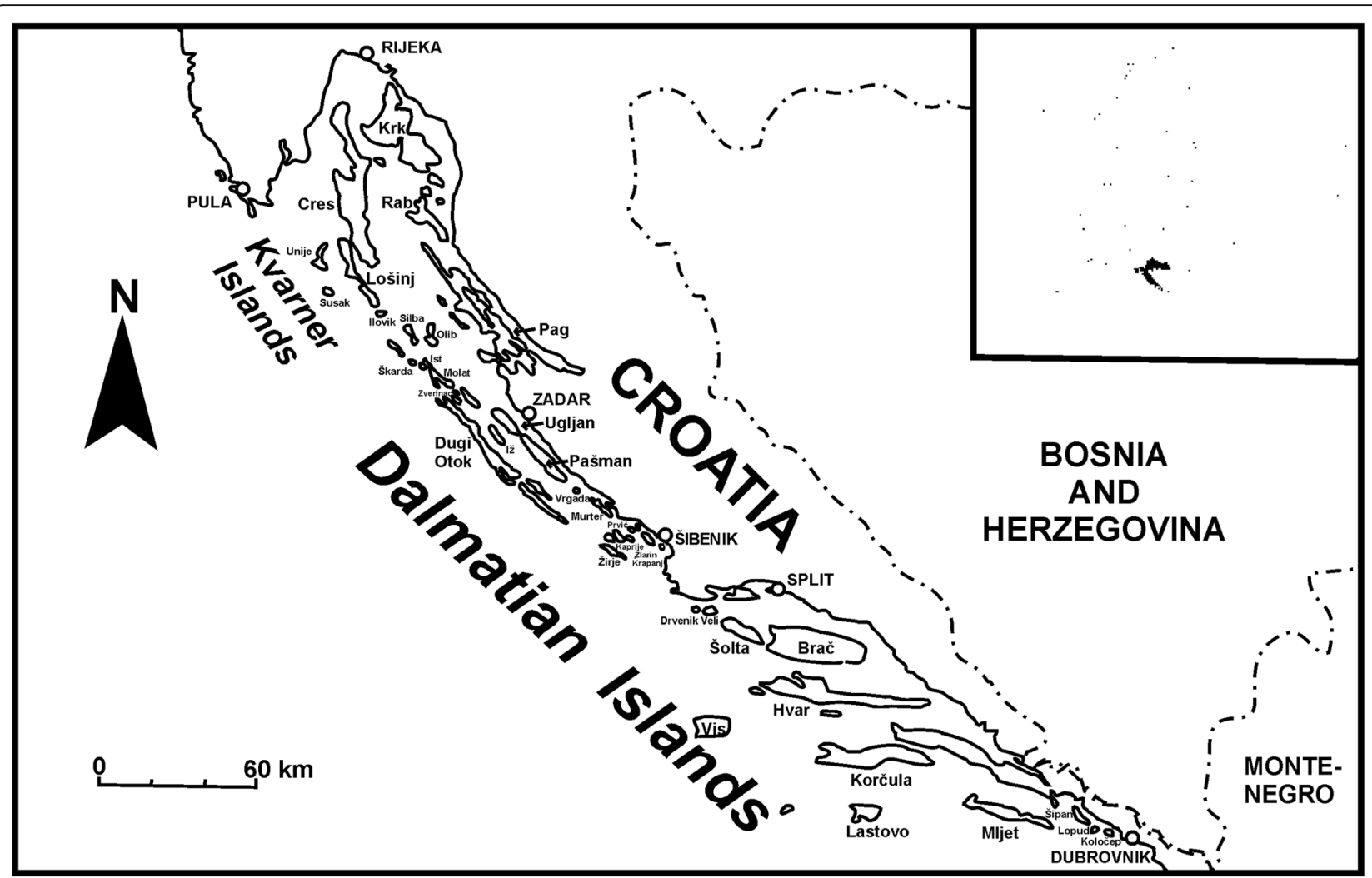

Fig. 1 Map of the Adriatic Sea and southern Croatia showing the studied islands (lower case font) and major cities (in capitals)

were performed in Croatian, the native language of the inhabitants. The interviews concerned different aspects of plant use, but here, we present data only on alcoholic beverages, and only with respondents who possessed such knowledge. We asked the general question, which plants (wild or cultivated) are used to flavour alcoholic drinks? We made efforts to cover the whole island evenly and recruit informants from as many different villages as possible. The informants were chosen from people who were born on the islands and had their ancestry there.

The number of species in the island floras was extracted from data gathered by Nikolić et al. [36], supplemented by the flora of Pašman [39], Vrgada [40], Olib [41], and Ist and Skarda [42]. The isolation of the island was measured as the distance $(\mathrm{km})$ between the mainland and the part of the island closest to it. The population data were taken from the Statistical Yearbook of 2015 [43].

Plants were identified using standard floras available in this area of Europe, including Nikolićs guide for the identification of Croatian flora [44], Pignatti's Flora of Italy [45] and the Flora Croatica Database [46]. Plant names were updated to be consistent with The Plant List [47]. Voucher specimens were collected on the islands where they were used, usually with the assistance of the respondents. For the place of deposition, see the "Availability of data and materials" section.

In some of our analyses, we divided the islands into five groups corresponding to their administrative location in five regions ('županija'): (1) Kvarner islands, (2) Zadar islands (North Dalmatian Islands), (3) Šibenik islands and (4) Split islands (Central Dalmatian Islands), and (5) Dubrovnik islands (South Dalmatian Islands). We grouped nearby islands, as there is usually cultural exchange between neighbouring islands, and the number of interviews from each island was very uneven.

Statistical analysis was performed using open-access PAST software [48]. The normality of the distribution of variables was tested with the Shapiro-Wilk test. All the variables had a normal distribution. A matrix of Pearson correlation coefficients for all pairs of variables was created (the variables used were number of species listed by an informant, gender of informant (male $=1$, female 0 ), age, area of the island, population, isolation from mainland, number of species in the vascular flora and longitude).

To visualise the similarity of species lists in the studied regions and in larger islands, we used a numeral taxonomy dendrogram obtained by clustering. We used the most common method of clustering, i.e. Unweighted 
Table 1 Islands where the interviews were conducted

\begin{tabular}{|c|c|c|c|c|c|c|}
\hline & Number of interviews & Area $\left(\mathrm{km}^{2}\right)$ & Population & Flora & Longitude (degrees E) & $\begin{array}{l}\text { Isolation (minimum } \mathrm{km} \\
\text { distance from mainland) }\end{array}$ \\
\hline \multicolumn{7}{|l|}{ Kvarner } \\
\hline Krk & 16 & 405 & 19,383 & 1170 & 14.61 & 0.8 \\
\hline Cres & 14 & 406 & 3079 & 1250 & 14.39 & 5 \\
\hline Rab & 14 & 86 & 9328 & 800 & 14.77 & 2 \\
\hline Lošinj & 13 & 74 & 7587 & 1300 & 14.43 & 29 \\
\hline llovik & 5 & 5 & 104 & n.d. & 14.55 & 56 \\
\hline Unije & 5 & 17 & 90 & 629 & 14.25 & 28 \\
\hline Susak & 3 & 4 & 188 & 400 & 14.30 & 41 \\
\hline \multicolumn{7}{|c|}{ Zadar Archipelago (Northern Dalmatia) } \\
\hline Pag & 15 & 284 & 9059 & 650 & 15.04 & 0.4 \\
\hline Pašman & 13 & 60 & 2845 & 629 & 15.34 & 2 \\
\hline Ugljan & 10 & 51 & 6049 & n.d. & 15.17 & 4 \\
\hline Dugi Otok & 9 & 113 & 1655 & 540 & 15.03 & 16 \\
\hline Molat & 9 & 22 & 207 & 308 & 14.83 & 17 \\
\hline Vrgada & 5 & 2 & 242 & 462 & 15.50 & 3 \\
\hline Iž & 3 & 16 & 557 & n.d. & 15.11 & 12 \\
\hline Olib & 3 & 26 & 147 & 465 & 14.79 & 25 \\
\hline Silba & 3 & 14 & 265 & 516 & 14.69 & 32 \\
\hline Ist & 2 & 10 & 202 & 438 & 14.76 & 20 \\
\hline Skarda & 1 & 4 & 0 & 237 & 14.71 & 32 \\
\hline Zverinac & 1 & 4 & 48 & n.d. & 14.92 & 18 \\
\hline \multicolumn{7}{|c|}{ Šibenik Archipelago (Central Dalmatia) } \\
\hline Murter & 6 & 18 & 5060 & 664 & 15.62 & 0.1 \\
\hline Žirje & 6 & 15 & 124 & 542 & 15.67 & 12 \\
\hline Kaprije & 5 & 7 & 143 & 278 & 15.71 & 5 \\
\hline Krapanj & 5 & 0.4 & 237 & 259 & 15.91 & 0.3 \\
\hline Prvić & 5 & 2 & 453 & 267 & 15.80 & 0.8 \\
\hline Zlarin & 5 & 8 & 276 & 444 & 15.85 & 2 \\
\hline \multicolumn{7}{|c|}{ Split Archipelago (Central Dalmatia) } \\
\hline Hvar & 16 & 297 & 11,077 & 1046 & 16.8 & 4 \\
\hline Šolta & 16 & 58 & 1700 & 267 & 16.31 & 15 \\
\hline Brač & 12 & 395 & 13,956 & 750 & 16.66 & 5 \\
\hline Vis & 11 & 90 & 3445 & 598 & 16.16 & 43 \\
\hline Drvenik Veli & 5 & & & n.d. & 16.15 & 2 \\
\hline \multicolumn{7}{|c|}{ Dubrovnik Archipelago (Southern Dalmatia) } \\
\hline Korčula & 22 & 271 & 15,522 & 858 & 16.93 & 1 \\
\hline Mljet & 14 & 98 & 1088 & 712 & 17.55 & 8 \\
\hline Lastovo & 10 & 41 & 792 & 678 & 16.87 & 26 \\
\hline Šipan & 5 & 16 & 436 & 570 & 17.88 & 2 \\
\hline Koločep (Kalamota) & 4 & 2 & 294 & 446 & 18.00 & 1 \\
\hline Lopud & 4 & 4 & 269 & 429 & 17.95 & 2 \\
\hline TOTAL & 295 & & & & & \\
\hline
\end{tabular}

n.d. no data available 
Pair Group Method with Arithmetic Mean (UPGMA), using Euclidean distance $[49,50]$.

\section{Results and discussion}

Altogether, 114 species of plants from 38 botanical families are involved in the production or flavouring of alcoholic drinks (Table 2, Figs. 2, 3, and 4). The largest families represented were Lamiaceae (19 species), Rosaceae (18), Asteraceae (12) and Rutaceae (6). The largest category is composed of wild plants (52 species, $46 \%$ of species). If we add the species which are gathered both from wild localities and from gardens (17 species), they constitute over half of the species list (Fig. 2). Fortythree species are exclusively cultivated, and only two species from one genus (coffee) are imported. The most important plant is of course grape (Vitis), which was not usually mentioned in interviews, regarded as being too obvious since wine is a basic everyday drink in southern Croatia. The remnants from the pressing of wine must, called 'pomace' in English (and drop in Croatian), are distilled into rakija. Rakija is a general term for any liquors distilled from fruits or their juices. Specifically, lozovača is the one made from grapes. Spirits have also been distilled from dried figs Ficus carica L. (the drink is called smokovača) and strawberry tree Arbutus unedo L. fruits (the latter species ceased to be used in 19501960s) or more rarely plum species šljivovica (Prunus spp.). The distillation of dried figs is still common on a very small scale (and the product is highly prized by alcohol gourmands). On the other hand, we have not found a single contemporary producer of strawberry tree distillate. At one time, this was so common that people from the Šibenik islands brought boats full of strawberry tree fruits and sold them in the Šibenik market.

The plants most commonly used to flavour drinks are fennel Foeniculum vulgare Mill., myrtle Myrtus communis L., sage Salvia officinalis L., rue Ruta graveolens L., juniper Juniperus oxycedrus L., carob Ceratonia siliqua L., walnut Juglans regia L., citrus fruit peel and leaves (mainly lemon Citrus limon (L.) Osbeck and bitter orange Citrus aurantium L.), fig Ficus carica L., bay Laurus nobilis L., rosemary Rosmarinus officinalis L., wormwood Artemisia absinthium L., petals of sweet smelling old rose garden varieties, mainly Rosa centifolia L., and mints (mainly Mentha piperita L. and M. spicata L.). Myrtle, walnut, rose petals and different cherry varieties and species (mainly Prunus cerasus, P. spinosa or $P$. avium) are used to make one-species sweetened liqueurs, which contain $20-40 \%$ alcohol. Other fruitbased single species sweet spirits are made of jujube Ziziphus jujuba L., nettle tree Celtis australis L., blackberry Rubus ulmifolius Schott, loquat Eriobotrya japonica (Thunb.) Lindl., dog rose Rosa canina L. and service tree Sorbus domestica L.
The remaining species are usually mixed. One kind of mixed-species spirit is called travarica ('trava' means 'herb'). The most common ingredients of this drink are fennel, sage, rue, juniper, carob, citrus fruit peel or leaves, bay, rosemary, wormwood, St. John's wort $H y$ pericum perforatum L., savory Satureja montana L., wild thyme (Thymus spp.), catmint Clinopodium nepeta (L.) Kuntze, marjoram Origanum majorana L., curry plant Helichrysum italicum (Roth) G. Don and lavender (Lavandula spp.).

The correlations between the number of species listed by an informant and the studied independent variables were very weak (Table 3 ). The strongest correlation was found with geographical longitude. Weak negative correlations were found for the age of informants and number of species in the local flora, i.e. younger people and people from more species-poor islands tended to quote more species! Men listed slightly more species than women, but the difference was small and insignificant.

Most of the common ingredients we found are used throughout the Adriatic Islands. On the other hand, some northwest-southeast trends can be detected for a few species, and the number of species per informant was lower in the Kvarner and Zadar archipelago than further south (Fig. 4). The largest number of species per interview was recorded for the Šibenik islands, but it was nearly identical for the Split and Dubrovnik islands as well (Fig. 4). In terms of species composition, clustering differentiated the archipelagos into two groups, one constituted by the Dubrovnik and Split islands and the other by the Kvarner, Zadar and Šibenik islands. Thus, the Šibenik islands have an intermediate character-people use as many species as they do further south, but the species composition is closer to the more northern Kvarner and Zadar islands (Fig. 5).

When each island was analysed separately the largest number of taxa was recorded in Korčula (49), Vis (39), Zlarin (37), Hvar (30) and Šipan (27). The clustering analysis revealed that Vis, Pag and Korčula were the least similar to other large islands (Fig. 6). During our interviews, we noticed a high level of experimentation. People often mentioned trying new plants in search of interesting flavours. As many as 42 taxa were mentioned by only one or two informants, which show a high level of idiosyncrasy in the species composition used for rakija. However, there are a few species which are both restricted to one island and constitute a salient part of local culture. One example is rakija flavoured with Artemisia caerulescens $\mathrm{L}$. made on Cres. The plant is nearly extinct now and the drink is made only by a few people on the island, as the species is difficult to find (this case is discussed in more detail later in this chapter). Another example is the use of grey rock-rose Cistus $\times$ incanus $\mathrm{L}$. and Rhamnus alaternus L. in travarica mixes, which is 
Table 2 The list of species used to make alcoholic drinks in the Adriatic Islands

\begin{tabular}{|c|c|c|c|c|c|c|c|c|c|c|c|c|c|}
\hline & Kvarner & $\begin{array}{l}\text { Zadar } \\
\text { Arch. }\end{array}$ & $\begin{array}{l}\text { Šibenik } \\
\text { Arch. }\end{array}$ & $\begin{array}{l}\text { Split } \\
\text { Arch. }\end{array}$ & $\begin{array}{l}\text { Dubrovnik } \\
\text { Arch. }\end{array}$ & Total & & Distribution & Local names* & 1 & 2 & 3 & $\begin{array}{l}\text { Medicinal } \\
\text { uses }\end{array}$ \\
\hline No. of interviews & 70 & 74 & 32 & 60 & 59 & 295 & & & & & & & \\
\hline Total no. of species & 48 & 53 & 47 & 68 & 60 & 114 & & & & & & & \\
\hline No. of species per interview & 2.7 & 3.9 & 5.7 & 5.8 & 5.5 & 4.5 & & & & & & & \\
\hline $\begin{array}{l}\text { Achillea millefolium L. } \\
\text { (Asteraceae) ZAGR39685 }\end{array}$ & & & & 1 & 1 & 2 & $\mathrm{cW}$ & $\begin{array}{l}\text { Korčula, } \\
\text { Šolta }\end{array}$ & stolisnik & $\mathrm{m}$ & r & $\mathrm{fl}$ & \\
\hline $\begin{array}{l}\text { Aesculus hippocastanum L. } \\
\text { (Sapindaceae) }\end{array}$ & 1 & 1 & & & & 2 & $c W$ & Lošinj & divlji marun & s & r & $\mathrm{fr}$ & $\begin{array}{l}\text { Only against } \\
\text { rheumatism }\end{array}$ \\
\hline $\begin{array}{l}\text { Agrimonia eupatoria L. } \\
\text { (Rosaceae) WA66922 }\end{array}$ & & 1 & & & & 1 & w & Dugi Otok & divlja melisa & $\mathrm{m}$ & r & ae & \\
\hline $\begin{array}{l}\text { Ajuga chamaepitys (L.) Schreb. } \\
\text { (Lamiaceae) WA66388 }\end{array}$ & & 1 & & & & 1 & w & Ugljan & trava iva & $\mathrm{m}$ & r & ae & \\
\hline Aloe sp. (Asphodelaceae) & & & & & 1 & 1 & $\mathrm{cW}$ & Lastovo & Aloe vera & s & r & I & \\
\hline $\begin{array}{l}\text { Aloysia citriodora Palau } \\
\text { (Verbenaceae) WA66484 }\end{array}$ & & & 1 & & 4 & 5 & $\mathrm{cW}$ & $\begin{array}{l}\text { mainly } \\
\text { Korčula, also } \\
\text { Zlarin }\end{array}$ & $\begin{array}{l}\text { alviz, alviza, } \\
\text { alviža, also: } \\
\text { bela luiđa ZL }\end{array}$ & $\mathrm{m}$ & r & ae & $\begin{array}{l}\text { Pregnant } \\
\text { women } \\
\text { should avoid } \\
\text { it }\end{array}$ \\
\hline $\begin{array}{l}\text { Anethum graveolens L. } \\
\text { (Apiaceae) WA66391 }\end{array}$ & & 4 & & & 1 & 5 & c & $\begin{array}{l}\text { Pag, Mljet, } \\
\text { Vrgada }\end{array}$ & $\begin{array}{l}\text { anita } \mathrm{PG} \text {, } \\
\text { kopar } \mathrm{ML} \text {, anis } \\
\text { VR }\end{array}$ & $\mathrm{m}$ & r & $\begin{array}{l}\text { ae, } \\
\text { l }\end{array}$ & \\
\hline $\begin{array}{l}\text { Arbutus unedo L. (Ericaceae) } \\
\text { WA66321 }\end{array}$ & 7 & 22 & 11 & 26 & 21 & 87 & w & $\begin{array}{l}\text { throughout, } \\
\text { more in the } \\
\text { south }\end{array}$ & $\begin{array}{l}\text { fruit: maginja, } \\
\text { magunja, } \\
\text { manjiga, } \\
\text { meginja, } \\
\text { mogunja, also: } \\
\text { plančići UN; } \\
\text { plant: planika }\end{array}$ & $\begin{array}{l}\mathrm{s} / \\
\mathrm{m}\end{array}$ & $\begin{array}{l}\mathrm{rb}, \\
\mathrm{r}\end{array}$ & $\mathrm{fr}$ & $\begin{array}{l}\text { Rakija used } \\
\text { to be } \\
\text { commonly } \\
\text { distilled from } \\
\text { it until } \\
\text { 1960s, now } \\
\text { only addition } \\
\text { to mixed } \\
\text { rakijas }\end{array}$ \\
\hline $\begin{array}{l}\text { Artemisia abrotanum L. } \\
\text { (Asteraceae) }\end{array}$ & & & & & 3 & 3 & c & $\begin{array}{l}\text { Lastovo, } \\
\text { Korčula }\end{array}$ & $\begin{array}{l}\text { broda KO, } \\
\text { srčano zelje LA }\end{array}$ & $\mathrm{m}$ & r & $\begin{array}{l}\text { ae, } \\
\text { l }\end{array}$ & $\begin{array}{l}\text { Only } \\
\text { medicinal to } \\
\text { induce } \\
\text { menstruation }\end{array}$ \\
\hline $\begin{array}{l}\text { Artemisia absinthium L. } \\
\text { (Asteraceae) WA66375 }\end{array}$ & 6 & 12 & 7 & 11 & 3 & 39 & $\mathrm{cW}$ & throughout & $\begin{array}{l}\text { pelin, also: } \\
\text { asinac, ašinac, } \\
\text { osjenač KO, } \\
\text { domaći pelin, } \\
\text { vrtlarski pelin } \\
\text { ŠO }\end{array}$ & $\begin{array}{l}\mathrm{m} / \\
\mathrm{s}\end{array}$ & $r$ & $\begin{array}{l}\text { ae, } \\
\text { l }\end{array}$ & $\begin{array}{l}\text { For } \\
\text { digestion, } \\
\text { stomach } \\
\text { ache, against } \\
\text { worms }\end{array}$ \\
\hline $\begin{array}{l}\text { Artemisia caerulescens L. } \\
\text { (Asteraceae) WA66473 }\end{array}$ & 2 & & & & & 2 & w & Cres & $\begin{array}{l}\text { morski pelin, } \\
\text { divlji pelin, } \\
\text { pelino }\end{array}$ & s & r & $\begin{array}{l}\text { ae, } \\
\text { l }\end{array}$ & $\begin{array}{l}\text { Against } \\
\text { worms }\end{array}$ \\
\hline $\begin{array}{l}\text { Arum italicum Mill. (Araceae) } \\
\text { WA66915 }\end{array}$ & & & & 1 & & 1 & w & Brač & žuminac & $\mathrm{m}$ & r & $?$ & \\
\hline Bellis perennis L. (Asteraceae) & 1 & & & & & 1 & w & Rab & divlja tratinčica & $\mathrm{s} /$ & r & ae & $\begin{array}{l}\text { Medicinal } \\
\text { rakija for } \\
\text { massaging } \\
\text { swollen legs }\end{array}$ \\
\hline $\begin{array}{l}\text { Calendula officinalis } \mathrm{L} \text {. } \\
\text { (Asteraceae) WA66413 }\end{array}$ & 1 & & & & & 1 & c & Rab & neven & $\begin{array}{l}\mathrm{s} / \\
\mathrm{m}\end{array}$ & r & $\mathrm{fl}$ & $\begin{array}{l}\text { Medicinal } \\
\text { rakija for } \\
\text { massaging } \\
\text { swollen legs }\end{array}$ \\
\hline $\begin{array}{l}\text { Carduus cf. nutans L. } \\
\text { (Asteraceae) }\end{array}$ & & 1 & & & & 1 & w & Molat & sikavica & $\mathrm{m}$ & $r$ & $f l$ & $\begin{array}{l}\text { Also against } \\
\text { stomach } \\
\text { ache }\end{array}$ \\
\hline
\end{tabular}


Table 2 The list of species used to make alcoholic drinks in the Adriatic Islands (Continued)

\begin{tabular}{|c|c|c|c|c|c|c|c|c|c|c|c|c|c|}
\hline & Kvarner & $\begin{array}{l}\text { Zadar } \\
\text { Arch. }\end{array}$ & $\begin{array}{l}\text { Šibenik } \\
\text { Arch. }\end{array}$ & $\begin{array}{l}\text { Split } \\
\text { Arch. }\end{array}$ & $\begin{array}{l}\text { Dubrovnik } \\
\text { Arch. }\end{array}$ & Total & & Distribution & Local names* & 1 & 2 & 3 & $\begin{array}{l}\text { Medicinal } \\
\text { uses }\end{array}$ \\
\hline $\begin{array}{l}\text { Celtis australis L. } \\
\text { (Cannabaceae) WA66311 }\end{array}$ & 1 & 4 & 3 & 5 & 5 & 18 & c & Throughout & $\begin{array}{l}\text { fafarinka, } \\
\text { fararikula, } \\
\text { kostanja, } \\
\text { kostela, } \\
\text { košćela, } \\
\text { košćila, } \\
\text { kopriva, } \\
\text { koprivić, } \\
\text { koprva }\end{array}$ & $\mathrm{s}$ & $r$ & $\mathrm{fr}$ & \\
\hline $\begin{array}{l}\text { Centaurium erythraea Rafn } \\
\text { (Gentianaceae) WA66924 }\end{array}$ & 1 & 2 & & 1 & & 4 & w & Throughout & kičica & $\mathrm{m}$ & $r$ & ae & $\begin{array}{l}\text { Stimulating } \\
\text { appetite }\end{array}$ \\
\hline $\begin{array}{l}\text { Ceratonia siliqua L. (Fabaceae) } \\
\text { WA66455 }\end{array}$ & 1 & 11 & 6 & 11 & 25 & 54 & c & $\begin{array}{l}\text { More in the } \\
\text { south }\end{array}$ & $\begin{array}{l}\text { karuba, korub, } \\
\text { rogač, also: VI } \\
\text { jubica }\end{array}$ & $\begin{array}{l}\mathrm{m} / \\
\mathrm{s}\end{array}$ & $r$ & $\mathrm{fr}$ & $\begin{array}{l}\text { Good for } \\
\text { stomach, } \\
\text { against } \\
\text { diarrhoea }\end{array}$ \\
\hline $\begin{array}{l}\text { Cistus } \times \text { incanus L. (Cistaceae) } \\
\text { WA66353 }\end{array}$ & & & & 4 & & 4 & w & Vis & $\begin{array}{l}\text { divji pelin, } \\
\text { carveni pelin }\end{array}$ & $\mathrm{m}$ & $r$ & I & \\
\hline $\begin{array}{l}\text { Cistus monspeliensis L. } \\
\text { (Cistaceae) WA66403 }\end{array}$ & & & & 1 & & 1 & & Vis & slipavi pelin & $\mathrm{m}$ & $r$ & I & \\
\hline $\begin{array}{l}\text { Cistus salviifolius L. (Cistaceae) } \\
\text { WA66449 }\end{array}$ & & & & 1 & & 1 & & Vis & pelin, bili pelin & $\mathrm{m}$ & $r$ & I & \\
\hline $\begin{array}{l}\text { Citrus } \times \text { aurantium } \mathrm{L} \text {. } \\
\text { (Rutaceae) WA71108 }\end{array}$ & & & & & 10 & 10 & $c$ & Only south & $\begin{array}{l}\text { divlja naranča, } \\
\text { gorka naranča, } \\
\text { limon, ljuta } \\
\text { naranča, } \\
\text { naranča }\end{array}$ & $\mathrm{m}$ & r & $\begin{array}{l}\mathrm{p}, \mathrm{l} \\
\mathrm{fr}\end{array}$ & \\
\hline $\begin{array}{l}\text { Citrus japonica Thunb. } \\
\text { (Rutaceae) }\end{array}$ & & & & 1 & & 1 & c & Drvenik Veli & kumkvat & $\mathrm{s} /$ & $r$ & $\mathrm{fr}$ & \\
\hline $\begin{array}{l}\text { Citrus limon (L.) Osbeck } \\
\text { (Rutaceae) WA66464 }\end{array}$ & 5 & 7 & 2 & 16 & 13 & 43 & $c$ & $\begin{array}{l}\text { Throughout } \\
\text { more in the } \\
\text { south }\end{array}$ & limon, limun & $\mathrm{s} /$ & $r$ & $\begin{array}{l}\mathrm{p}, \mathrm{l} \\
\mathrm{fr}\end{array}$ & $\begin{array}{l}\text { Fruit used to } \\
\text { make } \\
\text { limončelo or } \\
\text { fruit peel/ } \\
\text { leaves added } \\
\text { to mixed } \\
\text { travarica }\end{array}$ \\
\hline $\begin{array}{l}\text { Citrus reticulata Blanco } \\
\text { (Rutaceae) WA66333 }\end{array}$ & & 2 & 1 & 2 & 3 & 8 & c & $\begin{array}{l}\text { Throughout } \\
\text { more in the } \\
\text { south }\end{array}$ & $\begin{array}{l}\text { mandarina, } \\
\text { mandarinka, } \\
\text { (slatka) } \\
\text { naranča }\end{array}$ & $\mathrm{s} /$ & $r$ & $p, l$ & $\begin{array}{l}\text { Fruit used to } \\
\text { make a } \\
\text { single- } \\
\text { species } \\
\text { sweet li- } \\
\text { queur or } \\
\text { fruit peel/ } \\
\text { leaves added } \\
\text { to mixed } \\
\text { travarica }\end{array}$ \\
\hline $\begin{array}{l}\text { Citrus sinensis (L.) Osbeck } \\
\text { (Rutaceae) }\end{array}$ & & 1 & 1 & 13 & & 15 & $c$ & Throughout & naranča & $\mathrm{s} /$ & $r$ & $\begin{array}{l}\mathrm{p}_{1} \mathrm{l} \\
\mathrm{fr}\end{array}$ & $\begin{array}{l}\text { Fruit used to } \\
\text { make a } \\
\text { single- } \\
\text { species } \\
\text { sweet li- } \\
\text { queur or } \\
\text { fruit peel/ } \\
\text { leaves added } \\
\text { to mixed } \\
\text { travarica }\end{array}$ \\
\hline $\begin{array}{l}\text { Clinopodium nepeta (L.) } \\
\text { Kuntze (Lamiaceae) WA66943 }\end{array}$ & 1 & 5 & 1 & 4 & 4 & 15 & w & Throughout & $\begin{array}{l}\text { divlja menta, } \\
\text { divlja metvica, } \\
\text { menta, } \\
\text { metvica, also: } \\
\text { ošji mravinac } \\
\text { KO, šošenica VI }\end{array}$ & $\mathrm{m}$ & $r$ & ae & \\
\hline
\end{tabular}


Table 2 The list of species used to make alcoholic drinks in the Adriatic Islands (Continued)

\begin{tabular}{|c|c|c|c|c|c|c|c|c|c|c|c|c|c|}
\hline & Kvarner & $\begin{array}{l}\text { Zadar } \\
\text { Arch. }\end{array}$ & $\begin{array}{l}\text { Šibenik } \\
\text { Arch. }\end{array}$ & $\begin{array}{l}\text { Split } \\
\text { Arch. }\end{array}$ & $\begin{array}{l}\text { Dubrovnik } \\
\text { Arch. }\end{array}$ & Total & & Distribution & Local names* & 1 & 2 & 3 & $\begin{array}{l}\text { Medicinal } \\
\text { uses }\end{array}$ \\
\hline $\begin{array}{l}\text { Coffea arabica L. (Rubiaceae) } \\
\text { and C. liberica Hiern }\end{array}$ & & 1 & 2 & 1 & & 4 & $\mathrm{i}$ & Throughout & $\begin{array}{l}\text { mainly: kava, } \\
\text { also: kafa }\end{array}$ & $\mathrm{m}$ & $r$ & $\mathrm{~s}$ & \\
\hline $\begin{array}{l}\text { Cornus mas L. (Cornaceae) } \\
\text { WA66480 }\end{array}$ & 1 & & & & & 1 & w & Cres & drenjula & s & r & $\mathrm{fr}$ & \\
\hline $\begin{array}{l}\text { Crataegus azarolus L. } \\
\text { (Rosaceae) WA66306 }\end{array}$ & & & & & 1 & 1 & c & Korčula & glog & $\mathrm{s} /$ & $r$ & $\mathrm{fr}$ & \\
\hline $\begin{array}{l}\text { Crataegus monogyna Jacq. } \\
\text { (Rosaceae) WA66394 }\end{array}$ & 1 & 1 & & & & 2 & w & Krk, Pag & $\begin{array}{l}\text { glog crveni, } \\
\text { also: jabučica } \\
\text { PA }\end{array}$ & $\mathrm{s} /$ & r & $\mathrm{fr}$ & \\
\hline $\begin{array}{l}\text { Crocus biflorus ssp. weldenii } \\
\text { (Hoppe \& Fürnr.) K.Richt. } \\
\text { (Iridaceae) }\end{array}$ & & & & 1 & & 1 & w & Šolta & šafran & $\mathrm{m}$ & r & st & \\
\hline Crocus sativus L. (Iridaceae) & & & & & 5 & 5 & c & $\begin{array}{l}\text { Korčula, } \\
\text { Koločep }\end{array}$ & šafran & & $r$ & st & \\
\hline $\begin{array}{l}\text { Cydonia oblonga Mill. } \\
\text { (Rosaceae) }\end{array}$ & & & & 1 & & 1 & c & Vis & dunja & $\mathrm{m}$ & $r$ & $\mathrm{fr}$ & \\
\hline $\begin{array}{l}\text { Dioscorea communis (L.) } \\
\text { Caddick \& Wilkin } \\
\text { (Dioscoreaceae) ZAGR39307 }\end{array}$ & 1 & & & & & 1 & w & Krk & blušt & $\mathrm{s}$ & r & ae & $\begin{array}{l}\text { Only } \\
\text { medicinal, to } \\
\text { treat swollen } \\
\text { legs }\end{array}$ \\
\hline $\begin{array}{l}\text { Dittrichia viscosa (L.) Greuter } \\
\text { (Asteraceae) WA66302 }\end{array}$ & & 2 & & 2 & & 4 & w & $\begin{array}{l}\text { Dugi Otok, } \\
\text { Vis }\end{array}$ & $\begin{array}{l}\text { lipavi pelin, } \\
\text { pelin, tavari } \\
\text { bušinjak, } \\
\text { tovareći } \\
\text { bušinak }\end{array}$ & $\mathrm{m}$ & $r$ & ae & \\
\hline $\begin{array}{l}\text { Elymus repens (L.) Gould } \\
\text { (Poaceae) WA71140 }\end{array}$ & & & 1 & & & 1 & w & Prvić & pirika & $\mathrm{m}$ & $r$ & u & \\
\hline $\begin{array}{l}\text { Erica arborea L. (Ericaceae) } \\
\text { WA66432 }\end{array}$ & & 1 & & 2 & & 3 & w & $\begin{array}{l}\text { Dugi Otok, } \\
\text { Šolta, Vis }\end{array}$ & vris, vrisak & $\mathrm{m}$ & $r$ & ae & \\
\hline $\begin{array}{l}\text { Eriobotrya japonica (Thunb.) } \\
\text { Lindl. (Rosaceae) }\end{array}$ & 3 & 1 & & & 8 & 12 & c & throughout & $\begin{array}{l}\text { nespola, } \\
\text { nešpola, } \\
\text { nešpula }\end{array}$ & s & $r$ & $\mathrm{fr}$ & \\
\hline $\begin{array}{l}\text { Eucalyptus globulus Labill. } \\
\text { (Myrtaceae) }\end{array}$ & 1 & & & & & 1 & c & Lošinj & eukaliptus & $\mathrm{m}$ & r & I & \\
\hline Ficus carica L. (Moraceae) & 7 & 10 & 9 & 6 & 8 & 39 & c & Throughout & $\begin{array}{l}\text { divlja smokva, } \\
\text { smokva, also: } \\
\text { smokvenjak, } \\
\text { smokvenci LO, } \\
\text { smukva UG }\end{array}$ & $\mathrm{s} /$ & $\begin{array}{l}\mathrm{rb}, \\
\mathrm{r}\end{array}$ & $\mathrm{fr}$ & \\
\hline $\begin{array}{l}\text { Foeniculum vulgare Mill. } \\
\text { (Apiaceae) WA66401 }\end{array}$ & 17 & 24 & 15 & 31 & 19 & 106 & w & Throughout & $\begin{array}{l}\text { divji koromač, } \\
\text { komorač, } \\
\text { koromac, } \\
\text { koromač, } \\
\text { kromač, } \\
\text { morač, } \\
\text { koromacić }\end{array}$ & $\begin{array}{l}\mathrm{m} / \\
\mathrm{s}\end{array}$ & $r$ & ae & \\
\hline $\begin{array}{l}\text { Geranium macrorrhizum L. } \\
\text { (Geraniaceae) }\end{array}$ & & & & & 1 & 1 & c & Korčula & kanela & $\mathrm{m}$ & r & । & \\
\hline $\begin{array}{l}\text { Helichrysum italicum (Roth) } \\
\text { G.Don (Asteraceae) WA66460 }\end{array}$ & & 6 & 2 & 2 & 2 & 12 & w & Throughout & $\begin{array}{l}\text { cmilj, magriž, } \\
\text { smilj, smilje, } \\
\text { also: kadila PA }\end{array}$ & $\mathrm{m}$ & r & ae & \\
\hline $\begin{array}{l}\text { Hypericum perforatum L. } \\
\text { (Hypericaceae) WA66461 }\end{array}$ & & 7 & 4 & 7 & 3 & 21 & w & Throughout & $\begin{array}{l}\text { gospino cvićje, } \\
\text { gospina trava, } \\
\text { kantarion }\end{array}$ & $\mathrm{m}$ & r & $\begin{array}{l}\text { ae, } \\
\mathrm{fl}\end{array}$ & Stomachic \\
\hline $\begin{array}{l}\text { Juglans regia L. } \\
\text { (Juglandaceae) ZAGR39700 }\end{array}$ & 4 & 12 & 14 & 10 & 5 & 45 & $c$ & Throughout & orah, orih & s & r & $\mathrm{im}$ & Stomachic \\
\hline Juniperus macrocarpa Sm. & 10 & 2 & 8 & 4 & & 24 & w & Throughout & bujač, kleka, & $\mathrm{m}$ & r, & $\mathrm{fr}$ & Used widely, \\
\hline
\end{tabular}


Table 2 The list of species used to make alcoholic drinks in the Adriatic Islands (Continued)

\begin{tabular}{|c|c|c|c|c|c|c|c|c|c|c|c|c|c|}
\hline & Kvarner & $\begin{array}{l}\text { Zadar } \\
\text { Arch. }\end{array}$ & $\begin{array}{l}\text { Šibenik } \\
\text { Arch. }\end{array}$ & $\begin{array}{l}\text { Split } \\
\text { Arch. }\end{array}$ & $\begin{array}{l}\text { Dubrovnik } \\
\text { Arch. }\end{array}$ & Total & & Distribution & Local names* & 1 & 2 & 3 & $\begin{array}{l}\text { Medicinal } \\
\text { uses }\end{array}$ \\
\hline (Cupressaceae) WA66907 & & & & & & & & & $\begin{array}{l}\text { ljuskovac, } \\
\text { loskač, luskač, } \\
\text { luskavac, } \\
\text { luskavica, } \\
\text { luškač, } \\
\text { luškavica, } \\
\text { pucalinka, } \\
\text { pukomnjari, } \\
\text { smrič, smriče, } \\
\text { smričkalj, } \\
\text { smreka, smrijek, } \\
\text { smrika (fr } \\
\text { smričići), also: } \\
\text { slukovci RA, } \\
\text { šišarke [name } \\
\text { of fruits] ZL }\end{array}$ & & w & & $\begin{array}{l}\text { but in Cres } \\
\text { and Rab } \\
\text { made into a } \\
\text { kind of wine }\end{array}$ \\
\hline $\begin{array}{l}\text { Juniperus oxycedrus L. } \\
\text { (Cupressaceae) WA66332 }\end{array}$ & 17 & 9 & 7 & 17 & 9 & 59 & w & Throughout & $\begin{array}{l}\text { badljač, badovi } \\
\text { smric, badovi } \\
\text { smrik, bujač, } \\
\text { badžic, (divlja) } \\
\text { smreka, luskač, } \\
\text { luskovica, } \\
\text { luškač, pukinja, } \\
\text { smirčić, smrča, } \\
\text { smrekujići, } \\
\text { smrič, smrička, } \\
\text { smrijek, smrika, } \\
\text { smrjek, } \\
\text { smrjeka, } \\
\text { smriška, } \\
\text { šmrikuići, } \\
\text { šmirka, šmrika, } \\
\text { šmrkujić/ki, } \\
\text { šišarke [name } \\
\text { of fruits], also: } \\
\text { pomele CR }\end{array}$ & $\mathrm{m}$ & $\begin{array}{l}\mathrm{r} \\
\mathrm{w}\end{array}$ & $\mathrm{fr}$ & $\begin{array}{l}\text { Used widely, } \\
\text { but in Cres } \\
\text { and Rab } \\
\text { made into a } \\
\text { kind of wine }\end{array}$ \\
\hline $\begin{array}{l}\text { Juniperus phoenicea L. } \\
\text { (Cupressaceae) WA66326 }\end{array}$ & 1 & 1 & & 2 & & 4 & w & $\begin{array}{l}\text { llovik, Hvar, } \\
\text { Dugi Otok }\end{array}$ & $\begin{array}{l}\text { brika, gluhač, } \\
\text { luskavac, smrič }\end{array}$ & $\mathrm{m}$ & r & fr & \\
\hline $\begin{array}{l}\text { Laurus nobilis L. (Lauraceae) } \\
\text { WA66310 }\end{array}$ & 9 & 8 & 5 & 12 & 4 & 38 & $c W$ & Throughout & $\begin{array}{l}\text { javor, javorika } \\
\text { (fruit: javorčić), } \\
\text { lovor, lovorika, } \\
\text { also: laurano } \\
\text { CR }\end{array}$ & $\begin{array}{l}\mathrm{m} / \\
\mathrm{s}\end{array}$ & r & $\mathrm{l}, \mathrm{fr}$ & $\begin{array}{l}\text { Although } \\
\text { usually } \\
\text { leaves are } \\
\text { added to } \\
\text { mixed } \\
\text { travarica a } \\
\text { sweet } \\
\text { liqueur } \\
\text { made from } \\
\text { fruits is } \\
\text { made in Cres } \\
\text { and Lošinj }\end{array}$ \\
\hline $\begin{array}{l}\text { Lavandula } \times \text { intermedia } \\
\text { Emeric ex Loisel. (Lamiaceae) } \\
\text { WA71147 and L. angustifolia } \\
\text { Mill. WA66937 }\end{array}$ & & 2 & 1 & 2 & 3 & 8 & c & $\begin{array}{l}\text { Throughout } \\
\text { more in the } \\
\text { south }\end{array}$ & $\begin{array}{l}\text { lavanda, } \\
\text { levanda }\end{array}$ & $\mathrm{m}$ & r & ae & \\
\hline $\begin{array}{l}\text { Malus domestica Borkh. } \\
\text { (Rosaceae) WA66945 }\end{array}$ & 1 & 2 & 1 & & 1 & 5 & c & Throughout & $\begin{array}{l}\text { divlja jabuka, } \\
\text { jabuka }\end{array}$ & $\mathrm{m}$ & r & $\mathrm{fr}$ & \\
\hline $\begin{array}{l}\text { Matricaria chamomilla L. } \\
\text { (Asteraceae) WA66467 }\end{array}$ & & 2 & 1 & & 1 & 4 & $\mathrm{cW}$ & $\begin{array}{l}\text { Molat, } \\
\text { Zlarin, } \\
\text { Korčula }\end{array}$ & $\begin{array}{l}\text { kamilica, } \\
\text { kamomila }\end{array}$ & $\mathrm{m}$ & r & $\mathrm{fl}$ & \\
\hline Matthiola incana (L.) R.Br. & & & 1 & & & 1 & c & Žirje & domaća viola & $\mathrm{m}$ & r & $\mathrm{fl}$ & \\
\hline
\end{tabular}


Table 2 The list of species used to make alcoholic drinks in the Adriatic Islands (Continued)

\begin{tabular}{|c|c|c|c|c|c|c|c|c|c|c|c|c|c|}
\hline & Kvarner & $\begin{array}{l}\text { Zadar } \\
\text { Arch. }\end{array}$ & $\begin{array}{l}\text { Šibenik } \\
\text { Arch. }\end{array}$ & $\begin{array}{l}\text { Split } \\
\text { Arch. }\end{array}$ & $\begin{array}{l}\text { Dubrovnik } \\
\text { Arch. }\end{array}$ & Total & & Distribution & Local names* & 1 & 2 & 3 & $\begin{array}{l}\text { Medicinal } \\
\text { uses }\end{array}$ \\
\hline $\begin{array}{l}\text { Melissa officinalis L. } \\
\text { (Lamiaceae) WA66495 }\end{array}$ & & & 2 & 3 & 1 & 6 & c & Throughout & $\begin{array}{l}\text { čejena ljubica, } \\
\text { čelinja lubica, } \\
\text { ljubica, } \\
\text { matičnjak, } \\
\text { melisa, } \\
\text { pčelinja ljubica }\end{array}$ & $m$ & r & ae & \\
\hline $\begin{array}{l}\text { Mentha } \times \text { piperita } \mathrm{L} \text {. } \\
\text { (Lamiaceae) WA66402 and M. } \\
\text { spicata L. WA66348 }\end{array}$ & 2 & 4 & 4 & 4 & 11 & 25 & c & $\begin{array}{l}\text { Throughout } \\
\text { more in the } \\
\text { south }\end{array}$ & $\begin{array}{l}\text { divlja metica, } \\
\text { menta, menta } \\
\text { alkoholna, } \\
\text { menta divlja, } \\
\text { metvica, } \\
\text { minta, murtela, } \\
\text { also: guin } \\
\text { träva, kamilica } \\
\text { pitoma MU, } \\
\text { sarsa ŠO }\end{array}$ & $\mathrm{m}$ & $r$ & ae & \\
\hline $\begin{array}{l}\text { Micromeria graeca (L.) Benth. } \\
\text { ex Rchb. (Lamiaceae) }\end{array}$ & & & & 1 & & 1 & w & Vis & mažuron & $\mathrm{m}$ & r & ae & \\
\hline $\begin{array}{l}\text { Micromeria juliana (L.) Benth. } \\
\text { ex Rchb. (Lamiaceae) } \\
\text { WA66396 }\end{array}$ & & & & 2 & & 2 & w & Brač & bresina, čubar & $\mathrm{m}$ & r & ae & \\
\hline $\begin{array}{l}\text { Morus nigra L. (Moraceae) and } \\
\text { M. alba L. ZAGR39WA664 }\end{array}$ & & 1 & & 1 & 2 & 5 & c & Throughout & dud, murva & s & $r$ & $\mathrm{fr}$ & \\
\hline $\begin{array}{l}\text { Myrtus communis L. } \\
\text { (Myrtaceae) WA66307 }\end{array}$ & 15 & 29 & 7 & 22 & 28 & 101 & w & Throughout & $\begin{array}{l}\text { marka (fruit) } \\
\text { martovica } \\
\text { (plant), marta, } \\
\text { martići (fr), } \\
\text { martina, mirta, } \\
\text { markići (fr) } \\
\text { mirta (plant), } \\
\text { mrča, } \\
\text { mrčakinja, } \\
\text { mrčica, mrčka, } \\
\text { mrka, mrta, } \\
\text { mrtakinja, } \\
\text { mrtica, mrtina, } \\
\text { mrtovnica, } \\
\text { murta, murtići } \\
\text { (fr), smrške, } \\
\text { also: jurovika } \\
\text { KO, zoba LA }\end{array}$ & s & r & $\mathrm{fr}$ & \\
\hline $\begin{array}{l}\text { Ocimum basilicum L. } \\
\text { (Lamiaceae) WA71155 }\end{array}$ & & & 2 & 2 & 3 & 7 & c & Throughout & $\begin{array}{l}\text { bosiljak, } \\
\text { murtela }\end{array}$ & $\mathrm{m}$ & r & ae & \\
\hline Olea europaea L. (Oleaceae) & 3 & 4 & 1 & 2 & 1 & 11 & $c W$ & $\begin{array}{l}\text { A new } \\
\text { fashion }\end{array}$ & maslina & $\begin{array}{l}\mathrm{s} / \\
\mathrm{m}\end{array}$ & r & $\mathrm{fr}$ & \\
\hline $\begin{array}{l}\text { Origanum majorana L. } \\
\text { (Lamiaceae) WA66443 }\end{array}$ & & & 3 & 1 & 11 & 15 & c & $\begin{array}{l}\text { Korčula, } \\
\text { Mljet, Šolta, } \\
\text { Zlarin }\end{array}$ & $\begin{array}{l}\text { mažuran, } \\
\text { mažurana, } \\
\text { also: metvica } \\
\text { KO }\end{array}$ & $\mathrm{m}$ & $r$ & ae & \\
\hline $\begin{array}{l}\text { Origanum vulgare ssp. } \\
\text { viridulum (Martrin-Donos) } \\
\text { Nyman (Lamiaceae) WA71146 }\end{array}$ & 1 & & 1 & 2 & 3 & 7 & w & Throughout & $\begin{array}{l}\text { origano, } \\
\text { oregano, } \\
\text { mravinac }\end{array}$ & $\mathrm{m}$ & $r$ & ae & \\
\hline $\begin{array}{l}\text { Paliurus spina-christi Mill. } \\
\text { (Rhamnaceae) WA66316 }\end{array}$ & 1 & & 2 & & 1 & 4 & w & Throughout & drač, drača & $\mathrm{m}$ & $r$ & $\mathrm{im}$ & \\
\hline $\begin{array}{l}\text { Papaver rhoeas L. } \\
\text { (Papaveraceae) WA66381 }\end{array}$ & 1 & & & & & 1 & w & Krk & mak & $\mathrm{m}$ & $r$ & $\mathrm{fl}$ & \\
\hline $\begin{array}{l}\text { Parietaria judaica L. } \\
\text { (Urticaceae) WA66338 }\end{array}$ & 2 & 1 & & & & 3 & w & $\begin{array}{l}\text { Cres, Pag, } \\
\text { Rab }\end{array}$ & $\begin{array}{l}\text { lepek CR, šćir } \\
\text { PG, šćirenica } \\
\text { RB }\end{array}$ & s & $r$ & ae & $\begin{array}{l}\text { Only } \\
\text { medicinal for } \\
\text { kidneys, } \\
\text { bladder, } \\
\text { prostate, }\end{array}$ \\
\hline
\end{tabular}


Table 2 The list of species used to make alcoholic drinks in the Adriatic Islands (Continued)

\begin{tabular}{|c|c|c|c|c|c|c|c|c|c|c|c|c|c|}
\hline & Kvarner & $\begin{array}{l}\text { Zadar } \\
\text { Arch. }\end{array}$ & $\begin{array}{l}\text { Šibenik } \\
\text { Arch. }\end{array}$ & $\begin{array}{l}\text { Split } \\
\text { Arch. }\end{array}$ & $\begin{array}{l}\text { Dubrovnik } \\
\text { Arch. }\end{array}$ & Total & & Distribution & Local names* & 1 & 2 & 3 & $\begin{array}{l}\text { Medicinal } \\
\text { uses }\end{array}$ \\
\hline & & & & & & & & & & & & & veins \\
\hline $\begin{array}{l}\text { Pelargonium odoratissimum } \\
\text { (L.) L'Hér. (Geraniaceae) } \\
\text { WA71132 }\end{array}$ & & & & & 1 & 1 & c & Korčula & barbaroza & $\mathrm{m}$ & r & I & \\
\hline $\begin{array}{l}\text { Phillyrea latifolia L. (Oleaceae) } \\
\text { WA66446 }\end{array}$ & & & & 1 & & 1 & w & Vis & zelenika & $\mathrm{m}$ & r & $\mathrm{fr}$ & \\
\hline $\begin{array}{l}\text { Pimpinella anisum L. } \\
\text { (Apiaceae) }\end{array}$ & 1 & & & & 2 & 3 & c & $\begin{array}{l}\text { Cres, } \\
\text { Korčula, } \\
\text { Mljet }\end{array}$ & anis, aniš, aniž & $\begin{array}{l}\mathrm{s} / \\
\mathrm{m}\end{array}$ & r & $\mathrm{fr}$ & \\
\hline $\begin{array}{l}\text { Pinus halepensis Mill. } \\
\text { (Pinaceae) WA71148 }\end{array}$ & & & & & 1 & 1 & w & Korčula & bor & s & r & I & \\
\hline $\begin{array}{l}\text { Pinus pinea L. (Pinaceae) } \\
\text { WA66399 }\end{array}$ & & & & 1 & & 1 & c & Vis & pinj & s & r & I & \\
\hline $\begin{array}{l}\text { Pistacia lentiscus L. } \\
\text { (Anacardiaceae) WA66383 }\end{array}$ & 1 & & & 3 & & 4 & w & $\begin{array}{l}\text { Brač, Šolta, } \\
\text { Unije, Vis }\end{array}$ & $\begin{array}{l}\text { lonjstik UN, } \\
\text { smarška VS, } \\
\text { smrča ŠO, } \\
\text { smrčica BR }\end{array}$ & $\mathrm{m}$ & r & $\mathrm{fr}$ & \\
\hline $\begin{array}{l}\text { Plantago lanceolata L. } \\
\text { (Plantaginaceae) WA71162 }\end{array}$ & & & & 1 & & 1 & w & Korčula & trputac & $\mathrm{m}$ & r & । & \\
\hline $\begin{array}{l}\text { Prunus avium (L.) L. } \\
\text { (Rosaceae) WA66469 }\end{array}$ & 1 & 1 & & 1 & & 3 & c & $\begin{array}{l}\text { Cres, Ugljan, } \\
\text { Vis }\end{array}$ & $\begin{array}{l}\text { divja višnja VS, } \\
\text { trešnja CR, } \\
\text { trišnja UG }\end{array}$ & s & $r$ & $\mathrm{fr}$ & \\
\hline Prunus cerasus L. (Rosaceae) & & 8 & 6 & 5 & 5 & 24 & c & Throughout & $\begin{array}{l}\text { maraska, } \\
\text { maraška, višnja }\end{array}$ & s & $r$ & $\mathrm{fr}$ & \\
\hline $\begin{array}{l}\text { Prunus domestica L. } \\
\text { (Rosaceae) ZAGR39690 and P. } \\
\text { cerasifera Ehrh. }\end{array}$ & & & & 1 & 2 & 3 & $\mathrm{cW}$ & $\begin{array}{l}\text { Korčula, } \\
\text { Šolta }\end{array}$ & sliva & s & $\begin{array}{l}\mathrm{rb}, \\
\mathrm{r}\end{array}$ & $\mathrm{fr}$ & \\
\hline $\begin{array}{l}\text { Prunus dulcis (Mill.) D.A.Webb } \\
\text { (Rosaceae) }\end{array}$ & & 3 & 2 & 2 & 3 & 10 & c & Throughout & $\begin{array}{l}\text { badem, bajam, } \\
\text { mindel, } \\
\text { mindela, } \\
\text { mjendul }\end{array}$ & $\mathrm{m}$ & $r$ & pe & \\
\hline $\begin{array}{l}\text { Prunus mahaleb L. (Rosaceae) } \\
\text { WA66430 }\end{array}$ & & & & 1 & & 1 & w & Šolta & rašeljka & s & r & $\mathrm{fr}$ & \\
\hline $\begin{array}{l}\text { Prunus persica (L.) Batsch } \\
\text { (Rosaceae) WA66421 }\end{array}$ & 1 & & 3 & & 1 & 5 & $\mathrm{cW}$ & $\begin{array}{l}\text { Cres, } \\
\text { Lastovo, } \\
\text { Zlarin, Žirje }\end{array}$ & $\begin{array}{l}\text { breskva, } \\
\text { glodžanica Žl, } \\
\text { glodalica, } \\
\text { praska, voćka } \\
\text { ZL, praskva CR }\end{array}$ & s & r & $\mathrm{fr}$ & \\
\hline $\begin{array}{l}\text { Prunus spinosa L. (Rosaceae) } \\
\text { WA66367 }\end{array}$ & 3 & 6 & 2 & 1 & 1 & 13 & w & Throughout & $\begin{array}{l}\text { divlji trn, tarno, } \\
\text { torno, trn, } \\
\text { trnina, } \\
\text { trnjikula, } \\
\text { trnjovača, also: } \\
\text { brambula OL }\end{array}$ & s & $r$ & $\mathrm{fr}$ & \\
\hline $\begin{array}{l}\text { Punica granatum L. } \\
\text { (Lythraceae) WA66478 }\end{array}$ & 1 & & 3 & & 1 & 5 & $\mathrm{CW}$ & $\begin{array}{l}\text { Rab, } \\
\text { Krapanj, } \\
\text { Zlarin }\end{array}$ & $\begin{array}{l}\text { mogranj RB, } \\
\text { nar KR, šipak } \\
\text { ZL }\end{array}$ & $\mathrm{m}$ & r & pe & \\
\hline $\begin{array}{l}\text { Pyrus amygdaliformis Vill. } \\
\text { (Rosaceae) WA66904 }\end{array}$ & & 2 & & & & 2 & w & Pag & $\begin{array}{l}\text { divlja kruškica, } \\
\text { krušvica }\end{array}$ & s & r & $\mathrm{fr}$ & \\
\hline Pyrus communis L. (Rosaceae) & & & & & 2 & 2 & $c$ & $\begin{array}{l}\text { Korčula, } \\
\text { Šipan }\end{array}$ & kruška & s & r & $\mathrm{fr}$ & \\
\hline $\begin{array}{l}\text { Rhamnus alaternus L. } \\
\text { (Rhamnaceae) WA66920 }\end{array}$ & & & & 3 & & 3 & w & Vis & kokočika & $\mathrm{m}$ & r & $\mathrm{fr}$ & \\
\hline $\begin{array}{l}\text { Rosa canina L. (Rosaceae) } \\
\text { WA66309 and R. sempervirens } \\
\text { L. WA66323 }\end{array}$ & 4 & 3 & 1 & 1 & 1 & 10 & w & Throughout & $\begin{array}{l}\text { divlja ruža, } \\
\text { šipak, šipurak, } \\
\text { šepurika }\end{array}$ & $\mathrm{m}$ & r & $\mathrm{fr}$ & \\
\hline
\end{tabular}


Table 2 The list of species used to make alcoholic drinks in the Adriatic Islands (Continued)

\begin{tabular}{|c|c|c|c|c|c|c|c|c|c|c|c|c|c|}
\hline & Kvarner & $\begin{array}{l}\text { Zadar } \\
\text { Arch. }\end{array}$ & $\begin{array}{l}\text { Šibenik } \\
\text { Arch. }\end{array}$ & $\begin{array}{l}\text { Split } \\
\text { Arch. }\end{array}$ & $\begin{array}{l}\text { Dubrovnik } \\
\text { Arch. }\end{array}$ & Total & & Distribution & Local names* & 1 & 2 & 3 & $\begin{array}{l}\text { Medicinal } \\
\text { uses }\end{array}$ \\
\hline $\begin{array}{l}\text { Rosa centifolia L. (Rosaceae) } \\
\text { WA66941 }\end{array}$ & & 1 & 10 & 5 & 14 & 30 & c & $\begin{array}{l}\text { Commonly } \\
\text { but most } \\
\text { popular on } \\
\text { Korčula }\end{array}$ & $\begin{array}{l}\text { ruža, also: roza } \\
\text { iz kući KO }\end{array}$ & $\mathrm{s}$ & $r$ & $\mathrm{fr}$ & \\
\hline $\begin{array}{l}\text { Rosmarinus officinalis L. } \\
\text { (Lamiaceae) WA66366 }\end{array}$ & 3 & 9 & 4 & 12 & 9 & 37 & $\mathrm{cW}$ & $\begin{array}{l}\text { Throughout } \\
\text { more in the } \\
\text { south }\end{array}$ & $\begin{array}{l}\text { ružmarin, } \\
\text { lucmarin, also: } \\
\text { rusmarin, } \\
\text { ruzmarin, } \\
\text { zimorod, } \\
\text { zumorod, } \\
\text { zumrod, } \\
\text { zemorod HV }\end{array}$ & $\mathrm{m}$ & r & ae & \\
\hline $\begin{array}{l}\text { Rubus ulmifolius Schott } \\
\text { (Rosaceae) ZAGR39711 }\end{array}$ & 1 & 2 & 2 & & 10 & 15 & w & Throughout & $\begin{array}{l}\text { kupina, jagoda, } \\
\text { also: kupjena } \\
\text { Šl, kupjenica } \\
\text { LA, zrača KO }\end{array}$ & s & $\begin{array}{l}r_{1} \\
w\end{array}$ & $\mathrm{fr}$ & \\
\hline $\begin{array}{l}\text { Ruscus aculeatus L. } \\
\text { (Asparagaceae) WA66369 }\end{array}$ & 1 & & & & & 1 & w & Lošinj & leprinac & s & $r$ & $\mathrm{fr}$ & $\begin{array}{l}\text { Liqueur from } \\
\text { fruits }\end{array}$ \\
\hline $\begin{array}{l}\text { Ruta graveolens L. (Rutaceae) } \\
\text { WA66380 }\end{array}$ & 28 & 16 & 7 & 17 & 5 & 73 & $\mathrm{cW}$ & Throughout & $\begin{array}{l}\text { ruta, also: } \\
\text { rutnjak IŽ, } \\
\text { rutva } K R_{,}, L A \text {, } \\
\text { rutvica ŽZ, } \\
\text { trava ruta VI, } \\
\text { PA }\end{array}$ & $\begin{array}{l}\mathrm{s} / \\
\mathrm{m}\end{array}$ & r & ae & $\begin{array}{l}\text { To cure } \\
\text { infertility, } \\
\text { lack of } \\
\text { appetite, } \\
\text { protect from } \\
\text { evil eye, } \\
\text { against } \\
\text { worms }\end{array}$ \\
\hline $\begin{array}{l}\text { Salvia officinalis L. (Lamiaceae) } \\
\text { WA66459 }\end{array}$ & 6 & 13 & 10 & 28 & 17 & 74 & $\mathrm{cW}$ & Throughout & $\begin{array}{l}\text { kadulja, kaduja, } \\
\text { slavulja, } \\
\text { slavuja, also: } \\
\text { kuš CR, KR, LO, } \\
\text { pelin LO, ML, } \\
\text { Šl }\end{array}$ & $\mathrm{m}$ & r & $\mathrm{l}, \mathrm{fl}$ & \\
\hline $\begin{array}{l}\text { Salvia verbenaca } \mathrm{L} \text {. } \\
\text { (Lamiaceae) }\end{array}$ & & & & 1 & & 1 & w & Vis & $\begin{array}{l}\text { [no name } \\
\text { recorded] }\end{array}$ & $\mathrm{m}$ & $r$ & ae & \\
\hline $\begin{array}{l}\text { Sambucus nigra L. } \\
\text { (Adoxaceae) WA66415 }\end{array}$ & & & & 2 & 1 & 3 & c & Throughout & $\begin{array}{l}\text { bazga, also: } \\
\text { sambuk KO }\end{array}$ & s & r & $\mathrm{fl}$ & \\
\hline $\begin{array}{l}\text { Santolina chamaecyparissus L. } \\
\text { (Asteraceae) WA71111 }\end{array}$ & & & & 1 & & 1 & c & Vis & trovo od crvih & s & r & ae & $\begin{array}{l}\text { Only } \\
\text { medicinal } \\
\text { against } \\
\text { worms }\end{array}$ \\
\hline $\begin{array}{l}\text { Satureja montana L. } \\
\text { (Lamiaceae) WA66397 }\end{array}$ & & 8 & 1 & 7 & 3 & 19 & w & Throughout & $\begin{array}{l}\text { vrisak, also: } \\
\text { kidež ML, } \\
\text { meta DU, vris } \\
\text { BR }\end{array}$ & $\mathrm{m}$ & r & ae & \\
\hline $\begin{array}{l}\text { Satureja visianii Šilić } \\
\text { (Lamiaceae) WA66385 } \\
\text { (probably usually not } \\
\text { distinguished from S. } \\
\text { montana) }\end{array}$ & & & & & & & w & Korčula & vrisak & $\mathrm{m}$ & $r$ & ae & \\
\hline $\begin{array}{l}\text { Sedum cf. telephium L. s.l. } \\
\text { (Crassulaceae) WA66494 }\end{array}$ & & & & 1 & & 1 & c & Vis & žednjak & $\mathrm{m}$ & $r$ & । & \\
\hline $\begin{array}{l}\text { Sorbus domestica L. } \\
\text { (Rosaceae) WA66319 }\end{array}$ & & & 2 & 4 & 3 & 9 & $\mathrm{cW}$ & Throughout & $\begin{array}{l}\text { oskoruša, also: } \\
\text { oskorušica PG }\end{array}$ & s & r & $\mathrm{fr}$ & \\
\hline $\begin{array}{l}\text { Tanacetum balsamita L. } \\
\text { (Asteraceae) WA71150 }\end{array}$ & & & 2 & 1 & 1 & 4 & c & $\begin{array}{l}\text { Korčula, } \\
\text { Hvar, Zlarin }\end{array}$ & $\begin{array}{l}\text { kalober, } \\
\text { kaloper }\end{array}$ & $\mathrm{m}$ & $r$ & I & \\
\hline $\begin{array}{l}\text { Teucrium montanum L. } \\
\text { (Lamiaceae) }\end{array}$ & 1 & & & & 1 & 2 & w & Korčula, Krk & trava iva & $\mathrm{m}$ & $r$ & ae & \\
\hline $\begin{array}{l}\text { Teucrium polium L. } \\
\text { (Lamiaceae) WA66373 }\end{array}$ & & & & 2 & & 2 & w & Brač & trava iva & $\mathrm{m}$ & $r$ & ae & \\
\hline
\end{tabular}


Table 2 The list of species used to make alcoholic drinks in the Adriatic Islands (Continued)

\begin{tabular}{|c|c|c|c|c|c|c|c|c|c|c|c|c|c|}
\hline & Kvarner & $\begin{array}{l}\text { Zadar } \\
\text { Arch. }\end{array}$ & $\begin{array}{l}\text { Šibenik } \\
\text { Arch. }\end{array}$ & $\begin{array}{l}\text { Split } \\
\text { Arch. }\end{array}$ & $\begin{array}{l}\text { Dubrovnik } \\
\text { Arch. }\end{array}$ & Total & & Distribution & Local names* & 1 & 2 & 3 & $\begin{array}{l}\text { Medicinal } \\
\text { uses }\end{array}$ \\
\hline $\begin{array}{l}\text { Thymus longicaulis C.Presl } \\
\text { (Lamiaceae) WA71151 }\end{array}$ & 1 & 1 & & 6 & 8 & 16 & cW & $\begin{array}{l}\text { Brač, Cres, } \\
\text { Hvar, } \\
\text { Korčula, Pag, } \\
\text { Šipan, Vis }\end{array}$ & $\begin{array}{l}\text { majčina } \\
\text { dušica, } \\
\text { popunac, also: } \\
\text { poponac BR }\end{array}$ & $\mathrm{m}$ & $r$ & ae & \\
\hline $\begin{array}{l}\text { Urtica pilulifera L. (Urticaceae) } \\
\text { WA66441 and Urtica urens L. } \\
\text { (Urticaceae) WA66423 }\end{array}$ & & & & & 1 & 1 & w & Korčula & kopriva & $\mathrm{m}$ & r & I & \\
\hline $\begin{array}{l}\text { Vitex agnus-castus L. } \\
\text { (Lamiaceae) WA66905 }\end{array}$ & & 1 & & & & 1 & w & Pag & konopljika & $\mathrm{m}$ & $r$ & s & \\
\hline Vitis cf. vinifera L. (Vitaceae) & 3 & 2 & 1 & 2 & & 8 & c & Throughout & $\begin{array}{l}\text { grožđe (fruit), } \\
\text { vinova loza } \\
\text { (plant) }\end{array}$ & s & $\begin{array}{l}\text { rb, } \\
\text { r, } \\
w\end{array}$ & fr & \\
\hline $\begin{array}{l}\text { Ziziphus jujuba Mill. } \\
\text { (Rhamnaceae) WA66442 }\end{array}$ & 8 & 10 & 1 & 1 & 4 & 24 & c & $\begin{array}{l}\text { Throughout } \\
\text { more in the } \\
\text { north }\end{array}$ & $\begin{array}{l}\text { čičinka, } \\
\text { čičindra, } \\
\text { čičinda, žižula, } \\
\text { also: čičimak } \\
\text { Šl, čičindula } \\
\text { DU, zizula CR, } \\
\text { žužila UG }\end{array}$ & s & r & fr & \\
\hline
\end{tabular}

*Two capital letter abbreviations refer to particular islands and are made from the first two letters of their name, e.g. $Z L$ for Zlarin and $P A$ for Pašman, with an exception for PG Pag

1 -presence of other species: $\mathrm{m}$ - in mixed-species beverages, $\mathrm{s}$ - single species flavouring

2-type of use: $r$ - for rakija favouring, $r b$ - as base for rakija fermentation, $\mathrm{w}$ - as base for wine-type drinks (fermentation without distillation)

3-part of plant used: ae - aerial parts, fl - flowers, fr - fruit, im - immature fruit, I - leaf, pe - fruit peel, s - seeds, st - stigmas, u - underground parts

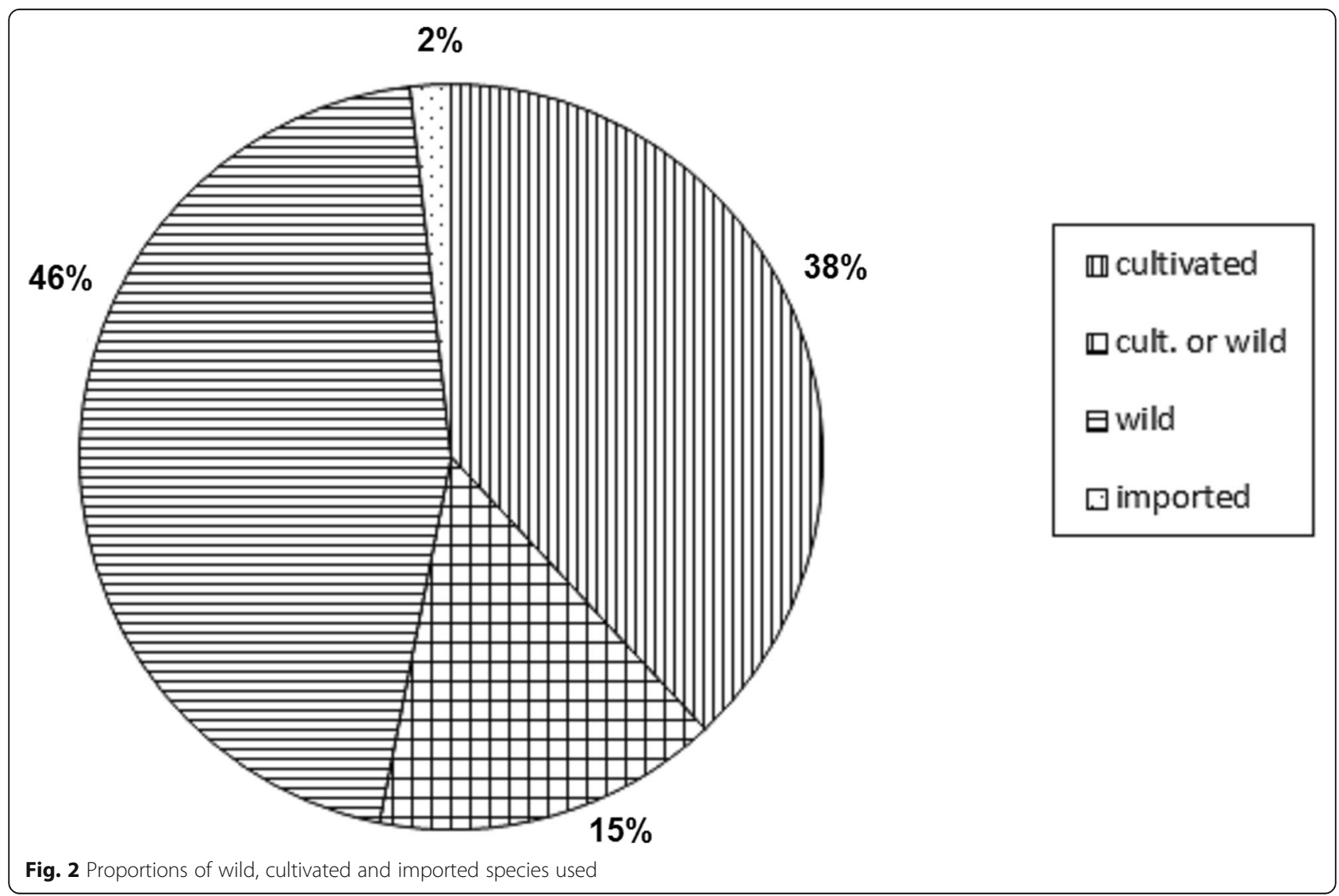




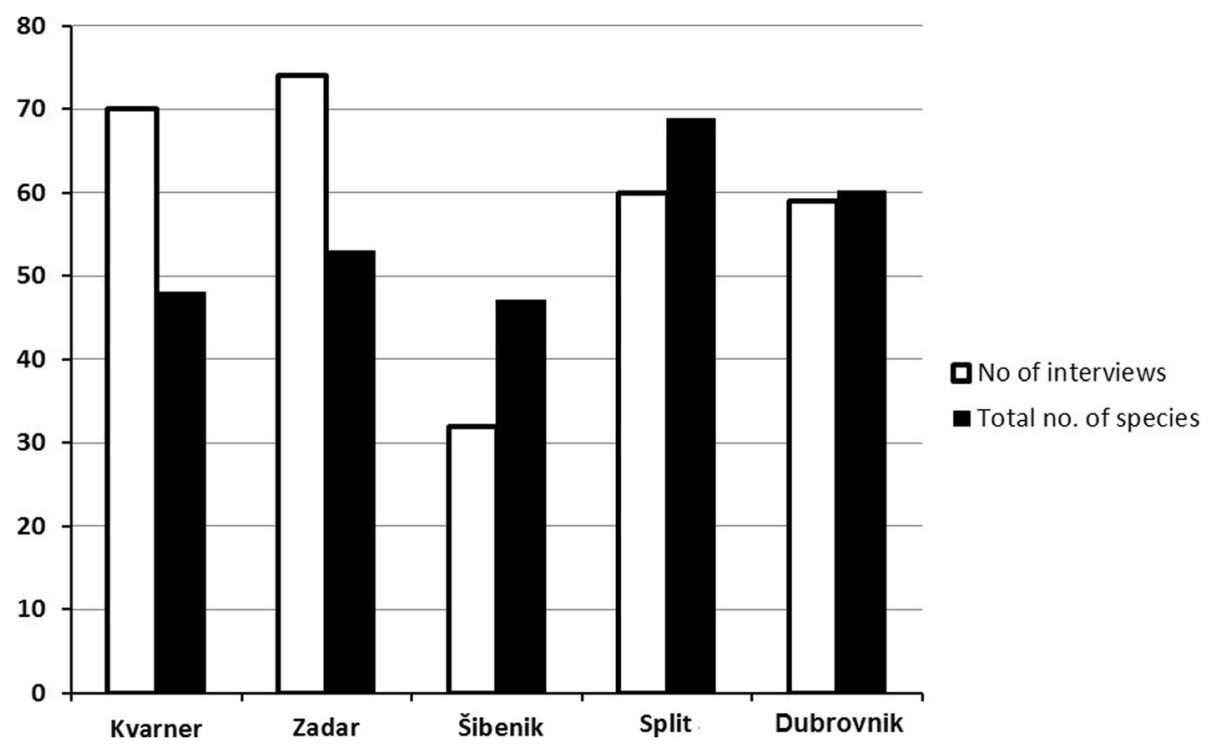

Fig. 3 Total number of species recorded in different parts of the Adriatic

restricted only to the island of Vis. These plants are very common on most of the islands [46], but are not used there. A similar situation occurs on Brač where the uses of Micromeria juliana (L.) Benth. ex Rchb. and felty germander Teucrium polium L. were recorded, but the species are found on other islands as well.

Citrus $\times$ aurantium L., bitter orange, is characteristic for the former Republic of Dubrovnik (now the
Dubrovnik region including the studied islands from this area), where it was introduced in the tenth century. It has a special place in gardens, summer houses and monasteries in the area of Dubrovnik. Bitter orange is often used in local gastronomy, as a spice and for medicinal purposes, and it is also an indispensable ingredient of the Dubrovnik 'travarica'. Interestingly, further north in other parts of Dalmatia (e.g. near Split), sweet orange

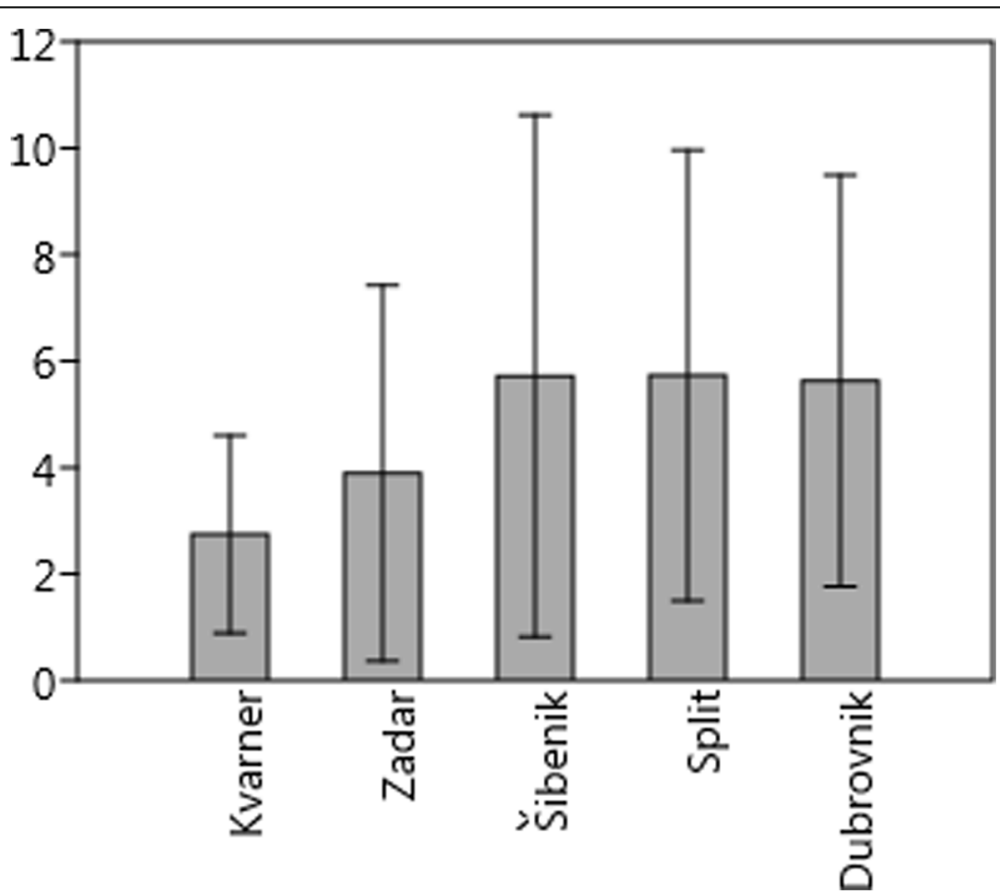

Fig. 4 Mean number (and standard deviation) of species mentioned per interview 
Table 3 Correlation matrix for the studied variables. Correlation coefficients are given in the lower left hand corner, the $p$ values in the upper right corner

\begin{tabular}{lllllllll}
\hline & Number of species & Male gender & Age & Area & Population & Flora & Longitude & Isolation \\
\hline Number of species & & 0.06846 & 0.01497 & 0.068294 & 0.33289 & 0.0023638 & $7.8724 \mathrm{E}-06$ & 0.83377 \\
Male gender & 0.10678 & & 0.014333 & 0.08595 & 0.10751 & 0.79029 & 0.10704 & 0.55088 \\
Age & $-0.14252^{*}$ & -0.14367 & & 0.39593 & 0.13228 & 0.31776 & 0.16676 & 0.87104 \\
Area & -0.10666 & 0.10067 & -0.049948 & & $5.5801 \mathrm{E}-61$ & $6.6006 \mathrm{E}-40$ & 0.48405 & $3.7934 \mathrm{E}-11$ \\
Population & -0.056766 & 0.094381 & -0.088442 & 0.77916 & & $4.8659 \mathrm{E}-32$ & 0.92627 & $1.4461 \mathrm{E}-11$ \\
Flora & $-0.18295^{*}$ & 0.016168 & -0.060801 & 0.68899 & 0.63263 & & $4.3672 \mathrm{E}-05$ & 0.10277 \\
Longitude & $0.25774^{*}$ & 0.094508 & -0.081271 & -0.041041 & -0.005429 & -0.24428 & -0.00011253 \\
Isolation & 0.012313 & -0.035043 & 0.0095575 & -0.37378 & -0.38116 & -0.098777 & -0.2237 & \\
\hline
\end{tabular}

* significant correlation between a dependent and independent variable, $p<0.05$

Citrus sinensis (L.) Osbeck is used more often than bitter orange. This difference has only a cultural or historical and not climatic explanation.

Among interesting plants occasionally used in rakija, we must mention lemon verbena, Aloysia citriodora Palau, which is used mainly on Korčula. According to local beliefs recorded in this study, the plant was brought by sailors to the island, directly from South America.

The recreational and digestive uses of plant flavoured alcohols are dominant, and they are rarely used for particular ailments. Digestive stomachic properties are generally attributed to mixed-species travarica. Out of all the herbal grappas, rue travarica is thought to be the most powerful. It is particularly used to increase appetite and prevent the evil eye ('evil eye' is a folk illness-it was commonly believed throughout Europe that a certain kind of person may hurt others, animals and crops, just by looking at them). The consumption or wearing of certain plants had a protective function against those with the 'evil eye' $[8,51]$. Other common stomachics are walnut (unripe fruit) and wormwood grappas. An

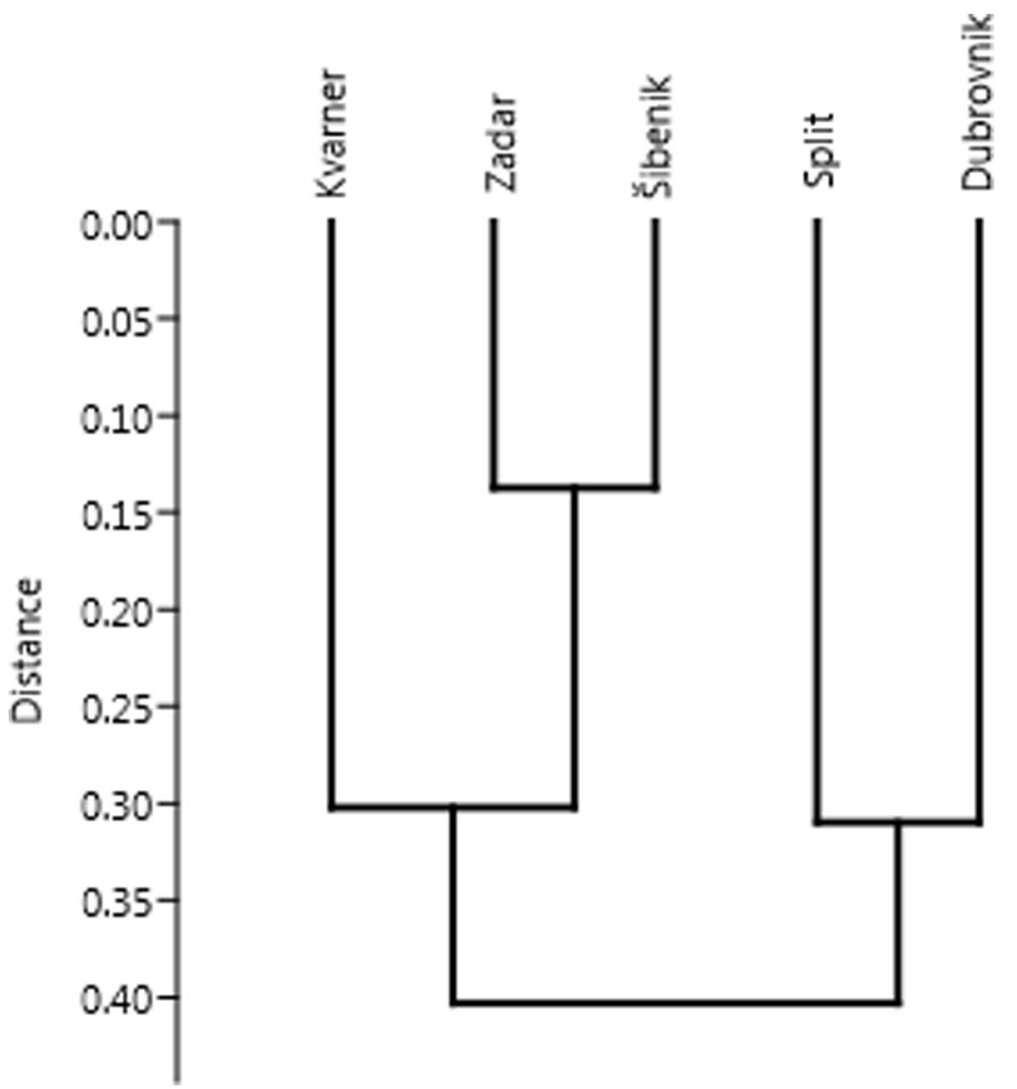

Fig. 5 Dendrogram of UPGMA clustering of regions based on the matrix of species used in them to flavour alcoholic drinks 


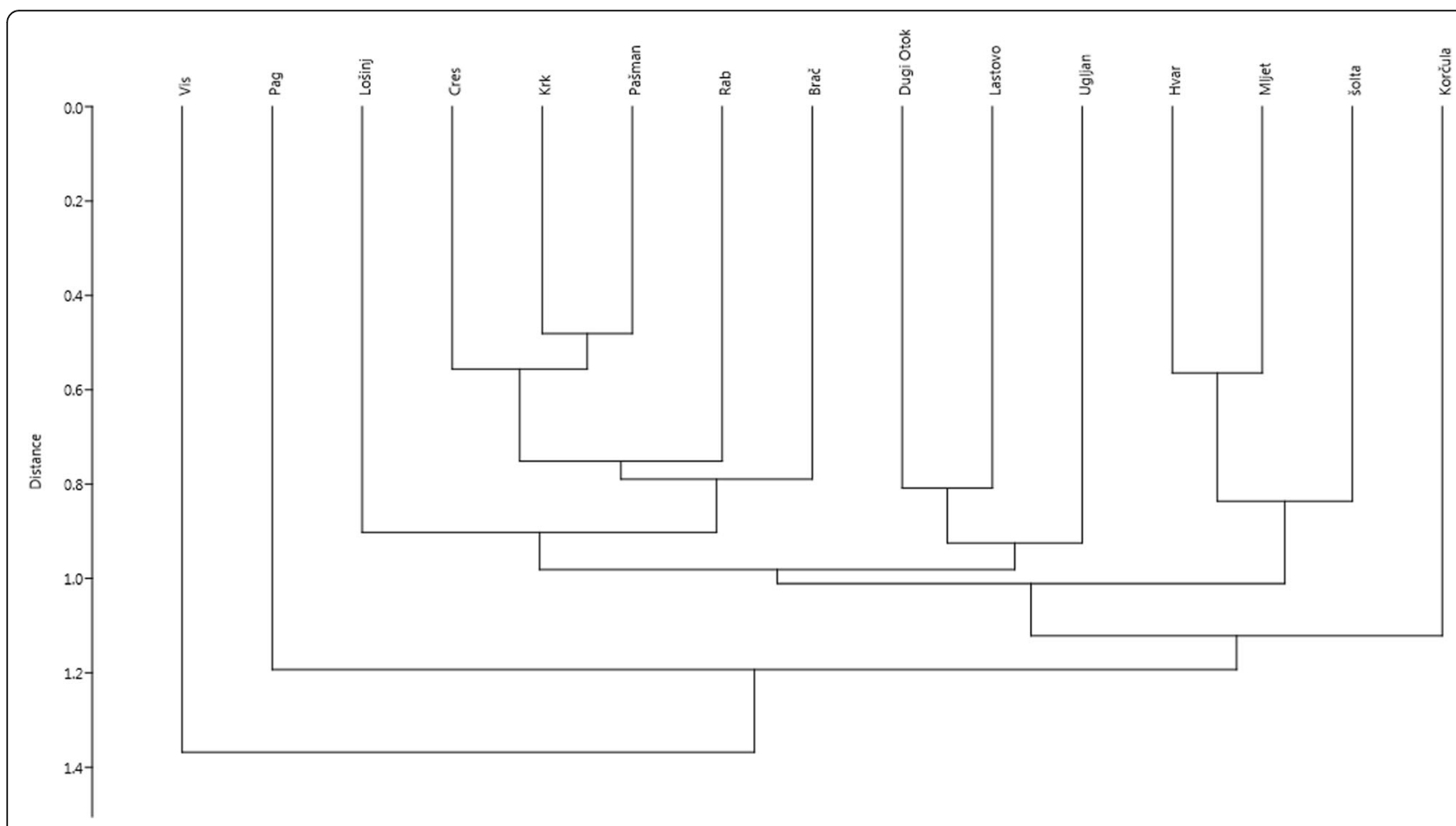

Fig. 6 Dendrogram of UPGMA clustering of the largest 15 islands based on the matrix of species used on them to flavour alcoholic drinks

interesting tradition persists in the Kvarner islands, where a sweet medicinal liqueur made from bay fruits is widely known.

Many of the species used to flavour alcoholic drinks are the same as those used on the other side of the Adriatic, in central Italy (sour cherries, walnuts, citrus fruits and many wild herbs such as sage) $[1,2,52]$. Thirty-two, i.e. nearly half of the wild plants recorded in the study, are also used for making liqueurs in Spain (including Catalonia) [7]. In Spain, similarly to the results of our study, Lamiaceae and Rosaceae are the families containing the largest number of plants used as liqueur ingredients. The similarity would be larger if it were not for the fact that in some cases different species from the same genus are used in the different countries (i.e. Thymus, Ruta, Pyrus). It is striking that in Croatia use of foreign (i.e. imported) ingredients in rakija is nearly nonexistent. Practically all the plants used for flavouring come from home gardens or the surrounding nature and are usually self-collected. Not a single species of imported spices is used (apart from coffee), in contrast to many other areas of Europe where spices such as star anise Illicium spp. or cloves Syzygium aromaticum (L.) Merrill \& Perry and cinnamon Cinnamomum spp. have been common ingredients of alcoholic drinks for a long time [15]. Strangely, the Venetians, who ruled the Croatian islands for hundreds of years, were some of the main traders of exotic spices [15]. In spite of this, the custom of combining them with alcohol did not permeate to the island culture. Locally grown Mediterranean spices, such as marjoram, anise and saffron, are also used sparingly. This avoidance of exotic imported spices in travarica goes along with the general trend of avoiding spices in south Croatian cuisine, particularly in Dalmatia [53]. Croatian cooking uses spices mainly for fish and game dishes (rosemary) or for preserving fruits and marinades (rosemary, bay leaf). Both rosemary and bay leaf are of local origin. Even native oregano and thyme species have not been, according to our respondents, used in cooking until recently. Making high alcohol spirits distilled from fruits must have a history stretching back a few hundred years in Croatia, and our respondents remember flavouring rakija with single species of mixed herbs since their childhood. However, they observed that making multi-species aromatic travarica has intensified as a result of the development of tourism since the 1960s. Before this time, single species flavouring was more widespread (rue, walnut, sour cherry, sage, myrtle). Another change has occurred in the way travarica is made. When people commonly distilled their rakija at home, herbs were added to the fermented fruits. Nowadays, they are more commonly added to bottles after the distillation process. In contrast to the thriving tradition of travarica, the use of local low-alcohol drinks from the pseudofruits of juniper (recorded on Rab and Cres, as well as on the mainland of Croatia in Istria [24]) has completely disappeared. 
Some new fashions may be observed, particularly making rakija with ripe olive fruits in it. The practice of adding exotic fruit species to rakija, mainly jujube and loquat, has also increased in frequency, though it was already present throughout the islands a few decades ago.

The incredible richness of species used in the Adriatic islands should be emphasised and treated as a local cultural heritage. In a very comprehensive review of plants used to make alcoholic drinks in the whole of Eastern Europe, 116 species were listed [6], whereas we recorded nearly as many as 114 in one part of one small country! In contrast, in a region of Italy as many as 46 species were recorded [2], which is still treated as a rich heritage. Comparable species richness was only observed in beer starters in a Shui minority area in Guizhou, China, where 102 species are used [12]. A large number of species used to produce home-made liqueurs is also used in Spain (including Catalonia), where at least 125 species of exclusively wild plants have been recorded [7], not taking into account cultivated species.

Although a rich tradition of flavouring strong spirits distilled from grape pomace is alive and even expanding, some culinary phenomena, such as the distillation of Arbutus unedo fruits, and making wine with Juniperus pseudo-fruits, are now extinct. These two beverages might be revived by local family farmers on a wave of general public interest in minor non-timber forest products [54]. It is worth emphasising that Juniperus spp. are spreading on land which once used to be grazed; thus, the utilisation of their fruits could increase local ecosystem services. The use of juniper beverages has been declining, in the Croatian islands as well as in northern Europe, with an exception of very few locations, e.g. Kurpie, Poland, where it has revived [9]. Madej et al. [9] claim that fermented juniper drinks have a very long history in Europe, of at least a few thousand years, based on archaeological findings from Denmark where ancient sediments of such a drink were found [55].

The common use of a large number of ingredients in rakija is probably a symptom of the gentrification of local gastronomic traditions. The term gentrification was first applied by the sociologist Ruth Glass in 1964 to ordinary, working-class areas of the city of London that became increasingly inhabited by middle-class people, artists etc. [56]. Now, the term is sometimes extended to cuisine as well. In contemporary times, we eagerly search for new ingredients and experiment with them [57]. Wild plants nowadays often become delicatessen as noted by the authors from the Iberian Peninsula [58]. They have also become an important part of menus in expensive restaurants [59]. In a tourism-oriented area of the Adriatic islands home-made spirits are often sold to visitors; thus, the pressure to impress tourists and provide an interesting product for them must have been an additional driving force towards experimentation with flavourings in alcohol. Interestingly, however, the ingredients used are nearly exclusively local, whether wild or grown. This is in contrast to other European countries, in which people often experiment with exotic spices. The Croatian islands formed part of the Venetian empire for a long period of time. The Venetians were some of the leading distilled alcohol producers in Europe. We are convinced that most of the flavouring ingredients we noted have a long history of local use in alcoholic drinks, but it used to be restricted to medicinal uses or used only by noblemen, and has only slowly become widespread and common. These species are usually used to make herbal teas as well; thus, it is likely that local people automatically switched from using the same species in herbal teas to using them in alcohol. The same likely trajectory was discussed for the popularisation of genepi in the western Alps. The name genepi is applied in the Western Alps (in Occitan, Franco-Provencal, and in French and Italian too) to diverse species of locally growing Alpine wormwood (esp. Artemisia genipi, A. glacialis, A. umbelliformis), which were once used locally as medicinal herbs, and later became part of a soughtafter alcoholic drink [60]. These species of Artemisia have been under threat of over-collection since the nineteenth century [61]. In our study, most of the plants used as flavouring are common or cultivated, apart from one which may be threatened. This is also an Artemisia species, bluish-leaved wormwood A. caerulescens. The species has a few dozen localities in coastal Croatia, restricted mainly to salt marshes, and is not very abundant [46]. The history of the interest in the utilisation of the species in the northern Adriatic is a long one [62]. It is only collected on the island of Cres, where it is known as morski pelin ('sea wormwood'), although the Venetianorigin name sandonego is also known. The local inhabitants are aware of the Venetian roots of the drink they make from it. According to our informants in Cres, it has been severely overharvested and practically destroyed by gathering and the development of tourist facilities on the shores, and it is now on the verge of extinction.

A. caerulescens and Satureja visianii Šilić seem to be the only relatively rare aromatic plant used. Other species from the impressive list of ingredients are common in the wild or commonly cultivated on the Croatian islands. Perhaps, this is what enabled a negative correlation between the number of species listed and the flora of the island (Table 3).

Starting the research, we expected a set number of species to be used in the multispecies rakijas. Certain numbers, such as 3,7 and their multiplications $(9,12$, 27) have positive connotations in European culture [8, 
63], and one of the authors observed that some people in northern Croatia use a set of 12 species for making rakija. However, here on the islands, no such customs were observed.

\section{Conclusions}

The study showed that the Croatian islands host a rich tradition of flavouring spirits. In terms of speciesrichness, this is, to the best of our knowledge, one of the longest lists of species used for flavouring alcoholic drinks ever recorded. Another interesting finding is the relic tradition of making a low-alcohol juniper beverage, mainly on the islands of the Kvarner archipelago.

\section{Acknowledgements}

We thank all the study participants who wanted to share their knowledge. Our special thanks go to Mirjana Jeričević for help in organising the interviews on the island of Korčula.

\section{Authors' contributions}

Łt contributed to the concept of the study and first draft of the paper. All the authors read and approved the final version of the paper and took part in the field study including interviewing and voucher specimen collection: $t$ t (30 islands, 261 interviews), IVK (22 islands, 174 interviews), MJD (6 islands, 69 interviews) and KD (5 islands, 49 interviews).

\section{Funding}

The research was financed by funds from the Ministry of Science and Higher Education, the National Science Centre in Poland (NCN) [2015/19/B/HS3/ 00471].

\section{Availability of data and materials}

The data matrix analysed during the current study is available upon request. Voucher specimens for species were deposited in the herbariums of Warsaw University (WAW) and the University of Zagreb (ZAGR).

\section{Ethics approval and consent to participate}

The research adhered to the local traditions for such research, the Code of Ethics of the International Society of Ethnobiology [64] and the American Anthropological Association Code of Ethics [65].

Prior oral informed consent was obtained from all study participants. No ethical committee permits were required. No permits were required to collect voucher specimens.

\section{Consent for publication}

Not applicable.

\section{Competing interests}

The authors declare that they have no competing interests.

\section{Author details}

${ }^{1}$ Institute of Biology and Biotechnology, University of Rzeszów, ul. Pigonia 1 , 35-310 Rzeszów, Poland. ${ }^{2}$ Institute for Adriatic Crops and Karst Reclamation, Put Duilova 11, 21000 Split, Croatia. Institute for Marine and Coastal Research, University of Dubrovnik, Kneza Damjana Jude 12, PO Box 83, 20000 Dubrovnik, Croatia. ${ }^{4}$ Department of Agricultural Botany, Faculty of Agriculture, University of Zagreb, Svetošimunska cesta 25, 10000 Zagreb, Croatia.

Received: 20 May 2019 Accepted: 9 October 2019

Published online: 05 November 2019

\section{References}

1. Egea T, Signorini MA, Bruschi P, Rivera D, Obón C, Alcaraz F, Palazón $J A$. Spirits and liqueurs in European traditional medicine: their history and ethnobotany in Tuscany and Bologna (Italy). J Ethnopharmacol. 2015;175:241-55.
2. Egea T, Signorini MA, Ongaro L, Rivera D, de Castro CO, Bruschi P. Traditional alcoholic beverages and their value in the local culture of the Alta Valle del Reno, a mountain borderland between Tuscany and EmiliaRomagna (Italy). J Ethnobiol Ethnomed. 2016;12(1):27.

3. Śliwińska M, Wiśniewska P, Dymerski T, Wardencki W, Namieśnik J. Authenticity assessment of the "Onisiówka" Nalewka liqueurs using twodimensional gas chromatography and sensory evaluation. Food Anal Meth. 2017;10(6):1709-20.

4. Galego L, Francisco V, Ratão I. Arbutus unedo L. fruit distillate from tradition to innovation. International congress on engineering and sustainability in the XXI century; 2017 Oct 11 (pp. 151-157). Cham: Springer; 2017.

5. Bhatt KC, Malav PK, Ahlawat SP. 'Jumin'a traditional beverage of Nocte tribe in Arunachal Pradesh: an ethnobotanical survey. Genet Res Crop Evol. 2018; 65(2):671-7

6. Sõukand R, Pieroni A, Biró M, Dénes A, Dogan Y, Hajdari A, Kalle R, Reade B, Mustafa B, Nedelcheva A, Quave CL, Łuczaj Ł. An ethnobotanical perspective on traditional fermented plant foods and beverages in Eastern Europe. J Ethnopharmacol. 2015;170:284-96.

7. Tardío J, Pardo-de-Santayana M, Morales R. Ethnobotanical review of wild edible plants in Spain. Bot J Linn Soc. 2006;152(1):27-71.

8. De Cleene M, Lejeune MC. Compendium of symbolic and ritual plants in Europe, vol 1 (trees and shrubs), vol 2 (herbs). Ghent: Mens \& Cultuur Uitgevers; 2003.

9. Madej T, Pirożnikow E, Dumanowski J, Łuczaj Ł. Juniper beer in Poland: the story of the revival of a traditional beverage. J Ethnobiol. 2014;34(1):84-103.

10. Austin GA. Alcohol in Western Society from Antiquity to 1800. A chronological history. Santa Barbara: ABC Clio; 1985.

11. Deka D, Sarma GC. Traditionally used herbs in the preparation of rice-beer by the Rabha tribe of Goalpara district, Assam. Indian J Tradit Know. 2010;9: 459-62.

12. Hong L, Zhuo J, Lei Q, Zhou J, Ahmed S, Wang C, Long Y, Li F, Long C. Ethnobotany of wild plants used for starting fermented beverages in Shui communities of Southwest China. J Ethnobiol Ethnomed. 2015;11(1):42.

13. Schoustra SE, Kasase C, Toarta C, Kassen R, Poulain AJ. Microbial community structure of three traditional Zambian fermented products: mabisi, chibwantu and munkoyo. PLoS One. 2013:8(5):e63948.

14. Hanousek Čiča K, Rupert M, Koczoń P, Derewiaka D, Gajdoš-Kljusurić J, Petravić-Tominac V, Mrvčić J, Stanzer D. Characterisation of flavour compounds in Biska-a herbal spirit produced with mistletoe. J Inst Brew. 2019;125(1):143-54.

15. Tonutti I, Liddle P. Aromatic plants in alcoholic beverages. A review. Flavour Fragr J. 2010;25(5):341-50.

16. Anderson $\mathrm{S}$, editor. Making medicines: a brief history of pharmacy and pharmaceuticals. London: Pharmaceutical Press; 2005.

17. Kremers E, Sonnedecker G. Kremers and Urdang's history of pharmacy. Madison: Amer. Inst. History of Pharmacy; 1986.

18. Łuczaj Ł, Zvonko Končić M, Miličević T, Dolina K, Pandža M. Wild vegetable mixes sold in the markets of Dalmatia (southern Croatia). J Ethnobiol Ethnomed. 2013;9:2.

19. Łuczaj $Ł$, Fressel $N$, Perković $S$. Wild food plants used in the villages of the Lake Vrana Nature Park (northern Dalmatia, Croatia). Acta Soc Bot Pol. 2013; 82:275-81.

20. Dolina K, Jug-Dujaković M, Łuczaj Ł, Vitasović-Kosić I. A century of changes in wild food plant use in coastal Croatia: the example of Krk and Poljica. Acta Soc Bot Pol. 2016;85(3):3508.

21. Dolina K, Łuczaj Ł. Wild food plants used on the Dubrovnik coast (southeastern (roatia). Acta Soc Bot Pol. 2014;83:175-81.

22. Łuczaj $Ł$, Dolina K. A hundred years of change in wild vegetable use in southern Herzegovina. J Ethnopharmacol. 2015;166:297-304.

23. Jug-Dujaković M, Łuczaj Ł. The contribution of Josip Bakićs research to the study of wild edible plants of the Adriatic coast: a military project with ethnobiological and anthropological implications. Slovak Ethnol. 2016;64:158-68.

24. Vitasović-Kosić I, Juračak J, Łuczaj Ł. Using Ellenberg-Pignatti values to estimate habitat preferences of wild food and medicinal plants: an example from northeastern Istria (Croatia). J Ethnobiol Ethnomed. 2017:13:31.

25. Varga F, Šolić I, Jug-Dujaković M, Łuczaj Ł, Grdiša M. The first contribution to the ethnobotany of inland Dalmatia: medicinal and wild food plants of the Knin area. Croatia Acta Soc Bot Pol. 2019;88(2):3622.

26. Pieroni A, Giusti ME. The remedies of the folk medicine of the Croatians living in Cíćarija, northern Istria. Coll Antropol. 2008;32(2):623-7. 
27. Pieroni A, Giusti ME, Münz H, Lenzarini C, Turković G, Turković A. Ethnobotanical knowledge of the Istro-Romanians of Žejane in Croatia. Fitoterapia. 2003;74(7-8):710-9.

28. Mrgić J. Aqua vitae-notes on geographies of alcohol production and consumption in the ottoman Balkans. Issues Ethnol Anthropol. 2017;12(4):1310-28.

29. Mrgić J. Wine or raki-the interplay of climate and society in early modern Ottoman Bosnia. Environ History. 2011;17(4):613-37.

30. Kerewsky-Halpern B. Rakija as ritual in rural Serbia. East Eur Quaterly. 1985; 18(4):481-94.

31. Miljić U, Puškaš V. Medicinal plants in Bermet, Serbian aromatic wine. Acta Agric Serb. 2012;17(34):83-92.

32. Keršek E. Ljekovito bilje u vinu i rakiji. Zagreb: VBZ doo; 2004.

33. Keršek E. Ljekovite biljne i voćne rakije. Zagreb: VBZ doo; 2008.

34. Łuczaj Ł, Jug-Dujaković M, Dolina K, Jeričević M, Vitasović-Kosić I. The ethnobotany and biogeography of wild vegetables in the Adriatic islands. J Ethnobiol Ethnomed. 2019;15:18.

35. MacArthur RH, Wilson EO. The theory of island biogeography. Princeton: Princeton University Press; 1967.

36. Nikolić T, Antonić O, Alegro AL, Dobrović I, Bogdanović S, Liber Z, Rešetnik I. Plant species diversity of Adriatic islands: an introductory survey. Plant Biosyst. 2008;142:435-45.

37. Trinajstić I. Biljne zajednice republike Hrvatske. Zagreb: Akademija šumarskih znanosti; 2008.

38. Kovačić S, Nikolić T, Ruščić M, Milović M, Stamenković V, Mihelj D, Jasprica N, Bogdanović S, Topić J. Flora jadranske obale i otoka - 250 najčešćih vrsta. Zagreb: Školska knjiga; 2008.

39. Pandža $M$, Milović M. Floristic researches of the island of Pašman, Croatia. In: Škvorc Ž, Franjić J, editors. 36th Meeting of Eastern Alpine and Dinaric Society for Vegetation Ecology - Book of Abstracts. Zagreb: Eastern Alpine and Dinaric Society for Vegetation Ecology; 2015. p. 43.

40. Pandža M, Milović M, Krpina V, Tafra D. Vaskularna flora vrgadskih otoka (Zadarski arhipelag, istočni Jadran). Nat Croat. 2011;20:97-116.

41. Milović M, Pandža M. Contribution to the study of Adriatic island flora: vascular plant species diversity in the Croatian Island of Olib. Nat Croat. 2016:25(1):25-54.

42. Milović M, Pandža M. Vaskularna flora otoka Ista i Škarde s pripadajućim otočićima i hridima. In: Otoci Ist i Škarda. Zadar: Sveučilište u Zadru; 2010. p. 149-86.

43. Ostroški L, ed. Statistički ljetopis Republike Hrvatske 2015 [Statistical Yearbook of the Republic of Croatia 2015]. Zagreb: Croatian bureau of statistics; 2015 http://wwwdzshr/Hrv_Eng/ljetopis/2015/sljh2015pdf Accessed 27 Aug 2018.

44. Nikolić T, editor. Flora Croatica 4 - Vaskularna flora Republike Hrvatske. Zagreb: Alfa d.d; 2019.

45. Pignatti S. Flora d'Italia. Bologna; 1982. p. Edagricole.

46. Flora Croatica Database. https://hirc.botanic.hr/fcd/ Accessed 15 Jan 2019.

47. The Plant List. http://theplantlist.org/ Accessed 15 Jan 2019.

48. Hammer $\varnothing$, Harper DAT, Ryan PD. PAST: paleontological statistics software package for education and data analysis. Palaeontol Electron. 2001;4:9.

49. Sokal R, Michener CA. Statistical method for evaluating systematic relationships. University of Kansas Science Bulletin. 1958;38:1409-38.

50. Bailey K. Typologies and taxonomies: an introduction to classification techniques. London-New Delhi: Sage Publications; 1994.

51. Pieroni A, Giusti ME. Ritual botanicals against the evil-eye in Tuscany, Italy. Econ Bot. 2002;56(2):201-3.

52. Motti $R$, Antignani $V$, Idolo M. Traditional plant use in the Phlegraean fields Regional Park (Campania, southern Italy). Hum Ecol. 2009;37(6):775.

53. Albala K. Food cultures of the world encyclopedia, vol. 2. Santa Barbara: ABC-CLIO; 2011.

54. Schunko C, Lechthaler S, Vogl CR. Conceptualising the factors that influence the commercialisation of non-timber Forest products: the case of wild plant gathering by organic herb farmers in South Tyrol (Italy). Sustainability. 2019; 11(7):2028.

55. McGovern PE, Hall GR, Mirzoian A. A biomolecular archaeological approach to 'Nordic grog'. Dan J Archaeol. 2013;2(2):112-31.

56. Glass RL. London: aspects of change. London: MacGibbon \& Kee; 1964.

57. Zukin S. Gentrification and cuisine. Consumption. 2001;3:418-39.

58. Reyes-García V, Menendez-Baceta G, Aceituno-Mata L, Acosta-Naranjo R, Calvet-Mir L, Domínguez P, Garnatje T, Gómez-Baggethun E, MolinaBustamante M, Molina M, Rodríguez-Franco R. From famine foods to delicatessen: interpreting trends in the use of wild edible plants through cultural ecosystem services. Ecol Econ. 2015;120:303-11.

59. Łuczaj Ł, Pieroni A, Tardío J, Pardo-de-Santayana M, Sõukand R, Svanberg I, Kalle R. Wild food plant use in 21 st century Europe, the disappearance of old traditions and the search for new cuisines involving wild edibles. Acta Soc Bot Pol. 2012;81(4):359-70.

60. Pieroni A, Giusti ME. Alpine ethnobotany in Italy: traditional knowledge of gastronomic and medicinal plants among the Occitans of the upper Varaita valley. Piedmont J Ethnobiol Ethnomed. 2009;5(1):32.

61. Chabert MA. Sur la conservation du Genépy dans nos Alpes. Bulletin de la Société Botanique de France. 1894;41(7):CLXXVI-IX.

62. Milhofer J. Botanische und chemische Untersuchungen an Artemisia caerulescens L. Acta Bot Croat. 1933;8(1):1-96.

63. Moszyński K. Kultura ludowa Słowian. Kraków: Polska Akademia Umiejętności; 1929.

64. International Society of Ethnobiology Code of Ethics (with 2008 additions). http://ethnobiology.net/code-of-ethics/ Accessed 10 Feb 2017.

65. American Anthropological Association Code of Ethics. http://www.aaanet. org/issues/policy-advocacy/upload/AAA-Ethics-Code-2009.pdf Accessed 10 Feb 2017.

\section{Publisher's Note}

Springer Nature remains neutral with regard to jurisdictional claims in published maps and institutional affiliations.
Ready to submit your research? Choose BMC and benefit from:

- fast, convenient online submission

- thorough peer review by experienced researchers in your field

- rapid publication on acceptance

- support for research data, including large and complex data types

- gold Open Access which fosters wider collaboration and increased citations

- maximum visibility for your research: over $100 \mathrm{M}$ website views per year

At $\mathrm{BMC}$, research is always in progress.

Learn more biomedcentral.com/submissions 